Check for updates

Cite this: RSC Adv., 2019, 9, 33853

\title{
Phosphotungstic acid as a novel acidic catalyst for carbohydrate protection and glycosylation $\dagger$
}

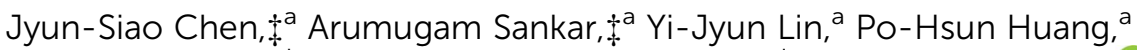 \\ Chih-Hsiang Liao, ${ }^{b}$ Shen-Shen $\mathrm{Wu}^{\mathrm{c}}{ }^{\mathrm{H}} \mathrm{H} \sin -\mathrm{Ru} \mathrm{Wu}^{\mathrm{d}}$ and Shun-Yuan Luo (iD *a
}

This work demonstrates the utilization of phosphotungstic acid (PTA) as a novel acidic catalyst for carbohydrate reactions, such as per-O-acetylation, regioselective $0-4,6$ benzylidene acetal formation, regioselective $\mathrm{O}-4$ ring-opening, and glycosylation. These reactions are basic and salient during the synthesis of carbohydrate-based bioactive oligomers. Phosphotungstic acid's high acidity and ecofriendly character make it a tempting alternative to corrosive homogeneous acids. The various homogenous acid catalysts were replaced by the phosphotungstic acid solely for different carbohydrate reactions. It can be widely used as a catalyst for organic reactions as it is thermally stable and easy to handle. In our work, the reactions are operated smoothly under ambient conditions; the temperature varies from $0{ }^{\circ} \mathrm{C}$ to room temperature. Good to excellent yields were obtained in all four kinds of reactions.

Received 8th August 2019

Accepted 11th October 2019

DOI: $10.1039 / c 9 r a 06170 c$

rsc.li/rsc-advances

It could be installed by using acid or base ${ }^{12 b}$ such as camphorsulfonic acid (CSA), ${ }^{12 c} p$-toluenesulfonic acid (TsOH), ${ }^{12 d}$ $\mathrm{SnCl}_{4}{ }^{12 e}$ and TCT. ${ }^{12 f}$ Nevertheless, the formation of 4,6-O-benzylidene acetals and its yields are limited by side-products and the requirement of severe acidic medium. ${ }^{12 g}$ Selective $O-4$ ring opening is also an important reaction of carbohydrates as it affords free hydroxyl group at C-4 position. This reaction proceeds in the presence of trifluoroacetic acid and triethylsilane $\mathrm{e}^{12 h, i}$ or sodium cyanoborohydride. ${ }^{13}$ However, this reaction provides low yield ${ }^{14 a}$ and sodium cyanoborohydride is dangerous, ${ }^{14 b}$ so new mediators for this reaction must be found to increase the yield. Finally, the glycosylation reaction requires an acid catalyst to build the oligosaccharides and it requires acid catalysts such as $\mathrm{FeCl}_{3} / \mathrm{C},{ }^{15 a} \mathrm{Cu}(\mathrm{OTf})_{2}{ }^{15 b} \mathrm{BF}_{3} \cdot \mathrm{OEt}_{2}$ or TMSOTf. ${ }^{15 c}$ However, no common acid catalyst for all of these reactions has been identified.

Hence, we would like to replace these different homogenous corrosive acid catalysts by using a sole acid catalyst for important reactions of carbohydrates. Furthermore, that acid catalyst must be eco-friendly, acidity must be strong enough, should not be corrosive and affordable. Fortunately, all of this requirements were fulfilled by the phosphotungstic acid. Thence, in this work, we have used phosphotungstic acid $\left(\mathrm{H}_{3}\left[\mathrm{P}\left(\mathrm{W}_{3} \mathrm{O}_{10}\right)_{4}\right]\right)$ catalyst for basic reactions namely per- $O$-acetylation, regioselective $O-4,6$ benzylidene acetal formation, regioselective $O-4$ reductive ring-opening, and glycosylation reactions. All of these reactions were underwent smoothly in the presence of PTA under ambient reaction condition.

PTA is a heteropolyacid, ${ }^{16 a}$ and a solid inorganic substance, ${ }^{16 b}$ it is following Keggin's structure. ${ }^{16 c}$ Phosphotungstic acid is non-toxic, ${ }^{17 a}$ eco-friendly, ${ }^{17 b}$ and the strongest heteropoly acid ${ }^{18 a}$ than common mineral acid such as $\mathrm{H}_{2} \mathrm{SO}_{4}, \mathrm{HCl}$, and

\footnotetext{
${ }^{a}$ Department of Chemistry, National Chung Hsing University, Taichung 402, Taiwan E-mail:syluo@dragon.nchu.edu.tw

${ }^{b}$ Taichung Municipal Feng Yuan Senior High School, Taichung 420, Taiwan 'National Hsinchu Girls' Senior High School, Hsinchu 300, Taiwan

${ }^{d}$ Instrumentation Center, National Tsing Hua University, MOST, Hsinchu 300, Taiwan $\dagger$ Electronic supplementary information (ESI) available. See DOI: 10.1039/c9ra06170c

\$ J.-S. Chen and A. Sankar contributed equally.
} 
$\mathrm{HNO}_{3} \cdot{ }^{18 b}$ It has a good chemical stability, thermal stability, reuse, and recycling. ${ }^{19-21}$ Phosphotungstic acid extensively used in well known organic reactions, including Prins cyclization, ${ }^{22 a}$ Claisen-Schmidt condensation, ${ }^{22 b}$ Schiff-bases synthesis, ${ }^{23}$ Pinacol-pinacolone rearrangements, ${ }^{24}$ Beckman rearrangements, ${ }^{25}$ bio-diesel synthesis in industries, ${ }^{26 a-c}$ and quinolone synthesis. $^{27}$

Neverthless, its impact in carbohydrate field is quite low and a few groups have been employed it as a catalyst for carbohydrate reactions. ${ }^{28 a, b}$ To the best of our knowledge, the applications of PTA in carbohydrates field is inadequate. Accordingly, our group extended the utilization of PTA to carbohydrates principle reactions, and obtaining good to excellent yields and will discuss in next part. These general reactions are depicted in Fig. 1.

\section{Results and discussion}

\section{Acetylation}

Table 1 presents the results of the phosphotungstic acidcatalyzed per-O-acetylation reaction. D-glucose 1a was used as the initial substrate to test phosphotungstic acid as a catalyst of per- $O$-acetylation. To test per- $O$-acetylation, the reaction was conducted under solvent-free conditions with D-glucose 1a, phosphotungstic acid (0.05 equiv.) and acetic anhydride (10 equiv.) in an atmosphere of nitrogen at room temperature. After 24 hours, the starting material was disappeared on the TLC plate and the desired product D-glucose pentaacetate $2 \mathrm{a}$ was obtained in $66 \%(\alpha / \beta=5 / 1)$ isolated yield (Table 1 , entry 1$)$. In an effort to increase the yield of the per-O-acetylated product, the amount of phosphotungstic acid was reduced to 0.01 equivalent. This change remarkably increased the yield of $2 \mathrm{a}$ to $88 \%(\alpha / \beta=5 / 1)$ without changing other reaction conditions (Table 1, entry 2). When 0.02 equivalent of phosphotungstic acid was used, the desired product $2 \mathbf{a}$ was afforded in good yield 93\% $(\alpha / \beta=5 / 1)$ (Table 1 , entry 3$)$.

Therefore, under the improved reaction conditions of per-Oacetylation by phosphotungstic acid, different substrates were screened. Unprotected sugars, such as D-galactose $\mathbf{1 b}, \mathrm{D}^{-}$ mannose 1c, methyl alpha-D-glucopyranoside 1d, D-xylose 1e and lactose $\mathbf{1 f}$ also participated in the reaction, which proceeded reaction smoothly under the same reaction conditions and producing $\mathrm{D}$-galactose pentaacetate $2 \mathbf{b}$ in $81 \%(\alpha / \beta=5 / 1)$, D-

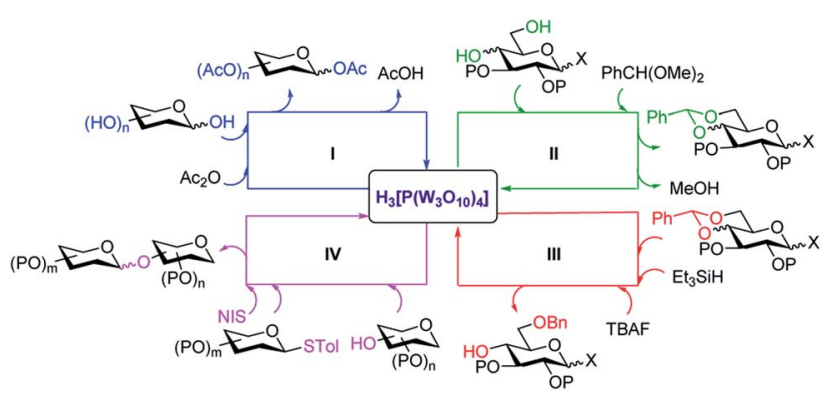

Fig. 1 Reactions of carbohydrates with phosphotungstic acid as the catalyst.
Table 1 Phosphotungstic acid catalyzed per-O-acetylation

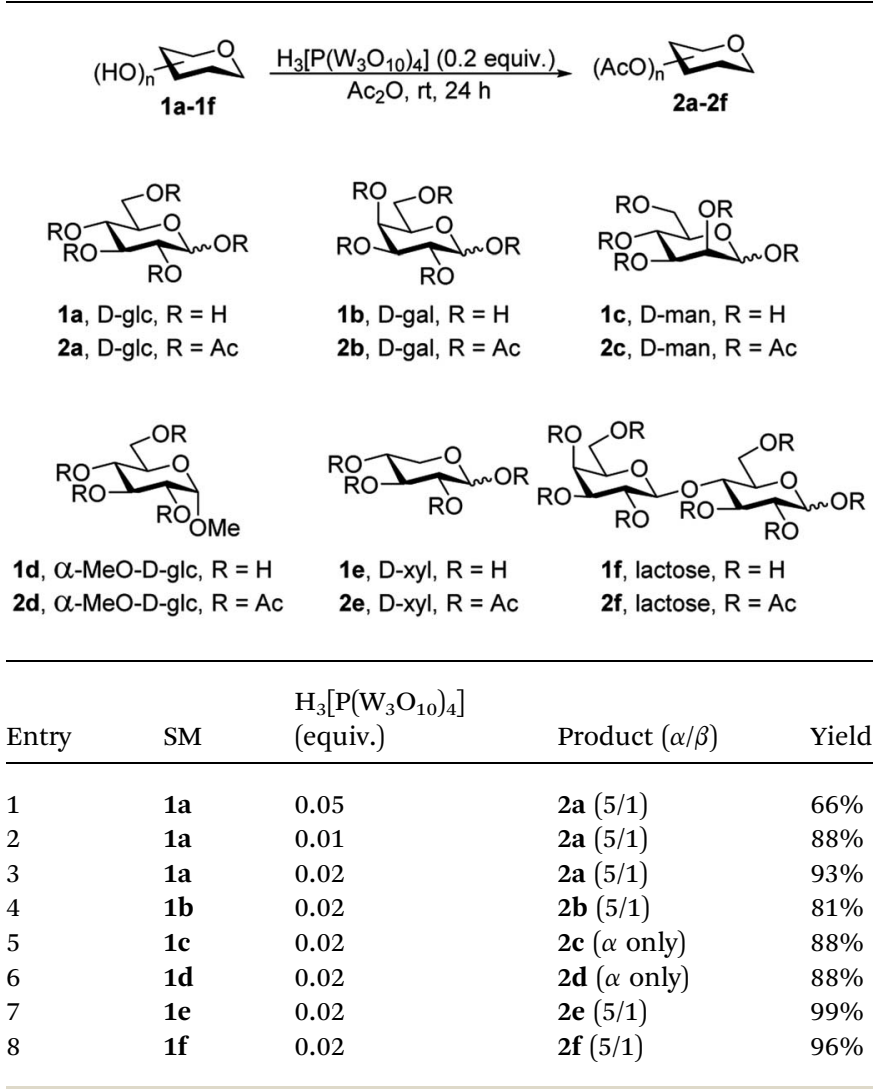

mannose pentaacetate $2 \mathrm{c}$ in $88 \%$ ( $\alpha$ only 1 ), $2 \mathrm{~d}$ in $88 \%$ ( $\alpha$ only), D-xylose tetraaacetate $2 \mathrm{e}$ in $99 \%(\alpha / \beta=5 / 1)$ and lactose octaacetate 2 f in $96 \%(\alpha / \beta=5 / 1)$ yields (Table 1 , entries $4-8)$. The various carbohydrates underwent reactions smoothly with acetic anhydride in the presence of phosphotungstic acid and the yields of acetylated products were excellent. These favorable findings help in solving problems such as the separation and corrosiveness of high-boiling-point liquid acids. Importantly, we developed a new method for per-O-acetylation with comparatively low cost PTA and it afforded good yield as $\mathrm{Cu}(\mathrm{OTf})_{2}$ and $\mathrm{HClO}_{4}$ wich are expensive catalysts. ${ }^{9 a, b}$

\section{Acetalization}

Phosphotungstic acid is used for the regioselective 4,6-O-benzylidene acetalization, as presented in Table 2 . The reaction of 4,6$O$-benzylidene acetalization started with 1d, phosphotungstic acid ( 0.5 equiv.), and benzaldehyde dimethyl acetal (2.0 equiv.) in acetonitrile. After 12 hours, the desired product 4 a was obtained in $71 \%$ isolated yield (Table 2 , entry 1 ). Initial attempts were made to find a suitable equivalent of phosphotungstic acid to enhance the yields of the desired product 4a. Notably, lowering the amount of phosphotungstic acid catalyst to 0.2 equivalent reduced the yield of $\mathbf{4 a}$ to $58 \%$ (Table 2, entry 2).

Therefore, the amount of phosphotungstic acid was not reduced any further. When 0.25 equivalent of $\mathrm{H}_{3}\left[\mathrm{P}\left(\mathrm{W}_{3} \mathrm{O}_{10}\right)_{4}\right]$ was used with 1d, the yield of product 4 a changed the yield remarkably to $69 \%$ (Table 2, entry 3 ). When the amount of catalyst was 
Table 2 Phosphotungstic acid catalyzed benzylidene acetal formation
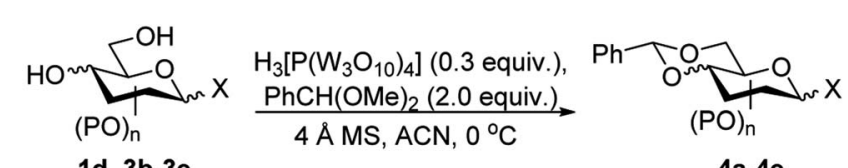

1d, $3 b-3 e$

$4 a-4 e$
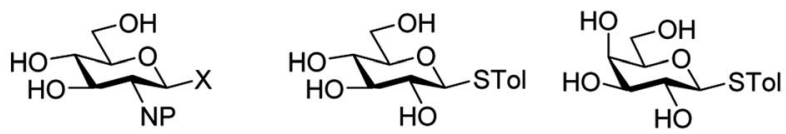

3b, $P=P h t h, X=\beta-S T$ ol

3d, D-Glc

3e, D-Gal

3c, $P=N_{2}, X=\beta$-OTBS
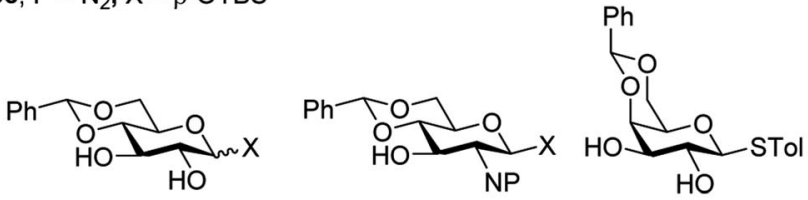

4a, D-Glc, $X=\alpha-\mathrm{OMe}$

4b, $P=P h t h, X=\beta-S T o l$

4e, D-Ga

4d, D-GIc, $X=\beta-$ STol

4c, $P=N_{2}, X=\beta$-OTBS

\begin{tabular}{lllll}
\hline & & $\begin{array}{l}\mathrm{H}_{3}\left[\mathrm{P}\left(\mathrm{W}_{3} \mathrm{O}_{10}\right)_{4}\right] \\
\text { (equiv.) }\end{array}$ & Product & Yield \\
\hline 1 & $\mathrm{SM}$ & 0.5 & $\mathbf{4 a}$ & $71 \%$ \\
2 & 1d & 0.2 & $\mathbf{4 a}$ & $58 \%$ \\
3 & $\mathbf{1 d}$ & $\mathbf{4 a}$ & $69 \%$ \\
4 & $\mathbf{1 d}$ & 0.25 & $\mathbf{4 a}$ & $72 \%$ \\
5 & $\mathbf{1 d}$ & $0.3^{a}$ & $\mathbf{4 a}$ & $69 \%$ \\
6 & $\mathbf{1 d}$ & $0.3^{b}$ & $\mathbf{4 b}$ & $84 \%$ \\
7 & 3b & 0.3 & $\mathbf{4 c}$ & $82 \%$ \\
8 & 3c & 0.15 & $\mathbf{4 d}$ & $65 \%$ \\
9 & 3d & 0.3 & $\mathbf{4 e}$ & $86 \%$
\end{tabular}

${ }^{a}$ The benzaldehyde dimethyl acetal was used 2.0 equivalents. ${ }^{b}$ The benzaldehyde dimethyl acetal was used 3.0 equivalents.

increased to 0.3 equivalent, the desired product $4 \mathrm{a}$ was obtained in a slightly higher yield $72 \%$ (Table 2 , entry 4 ). Ultimately, 0.3 equivalent of phosphotungstic acid was determined to be the optimal amount for the regioselective 4,6-O-benzylidene acetalization reaction. Increasing the amount of benzaldehyde dimethyl acetal to 3.0 equivalent did not significantly affect the yield of $\mathbf{4 a}$, which remained $69 \%$ (Table 2, entry 5). Therefore, various substrates were examined under optimized reaction conditions. $\beta$-Thio-D-glucoside derivative $\mathbf{3 b}$ was reacted with benzaldehyde dimethyl acetal in presence of phosphotungstic acid. The starting material was consumed in 6 hours and $4 \mathbf{b}$ was obtained in $84 \%$ yield (Table 2, entry 6). After $\mathbf{3 c}$ underwent the reaction, and the desired product $\mathbf{4 c}$ was obtained in $82 \%$ yield (Table 2, entry 7 ). $3 \mathbf{d}$ and $3 \mathrm{e}$ underwent the reaction, and afforded $4 \mathrm{~d}$ yield in $65 \%$ and $4 \mathbf{e}$ in $86 \%$ yield, respectively (Table 2 , entries $8-9$ ). It has produced better yield than PTSA ${ }^{12 d}$ catalyst and similar yield as CSA. PTA only required less amount than CSA to bring better yield of benzylidene acetal.

\section{Regioselective reductive ring opening of benzylidene acetals}

Following the successful per- $O$-acetylation and regioselective benzylidene ring formation, the $O-4$ selective ring opening reaction was considered. First, methyl 2,3-di-O-benzyl-4,6-Obenzylidene- $\alpha$-D-glucopyranoside $\mathbf{5 a}$ was used as the optimal substrate. At the outset, 5a, phosphotungstic acid (0.05 equiv.) and triethylsilane (11.0 equiv.) were used in DCM at $0{ }^{\circ} \mathrm{C}$. The expected product 6a was formed and its $\mathrm{O}-4$ silylated side product also observed on the TLC plate. To cleave the silyl group at the $O-4$ position, the reaction mixture was treated with tetra$n$-butyl ammonium fluoride (TBAF, 11.0 equiv.) and acetic acid (AcOH, 11.0 equiv.) at room temperature for 30 minutes, providing $\mathbf{6 a}$ in $31 \%$ isolated yield (Table 3 , entry 1 ). For further optimization, the amount of $\mathrm{H}_{3}\left[\mathrm{P}\left(\mathrm{W}_{3} \mathrm{O}_{10}\right)_{4}\right]$ was increased to 0.1 equivalent, significantly changing the yield of $6 \mathbf{a}$ to $80 \%$ (Table 3 , entry 2). Further increasing the amount of catalyst to 0.2 equivalent did not have a considerable effect in $79 \%$ yield (Table 3 , entry 3$)$.

Based on these results, the various substrate for the $O-4$ ring opening reaction was investigated. The $\beta$-thio $\mathrm{D}$-glucoside derivatives $\mathbf{5 b}$ and $\mathbf{5 c}$ underwent the reaction readily, giving $\mathbf{6 b}$ in $82 \%$ yield and $\mathbf{6 c}$ in $86 \%$ yield, respectively (Table 3, entries $4-5)$. The $\beta$-thio-D-galactose derivative $5 \mathbf{d}$ gave $\mathbf{6 d}$ in a similar yield $79 \%$ (Table 3 , entry 6). Importantly, after the completion of the reaction of $5 \mathbf{e}, \mathrm{TBAF}$ and $\mathrm{AcOH}$ were used to cleave its $O-4$ silylated group. However, under TBAF and $\mathrm{AcOH}$ condition,

Table 3 Phosphotungstic acid catalyzed regio-selective $0-4$ ring opening reactions

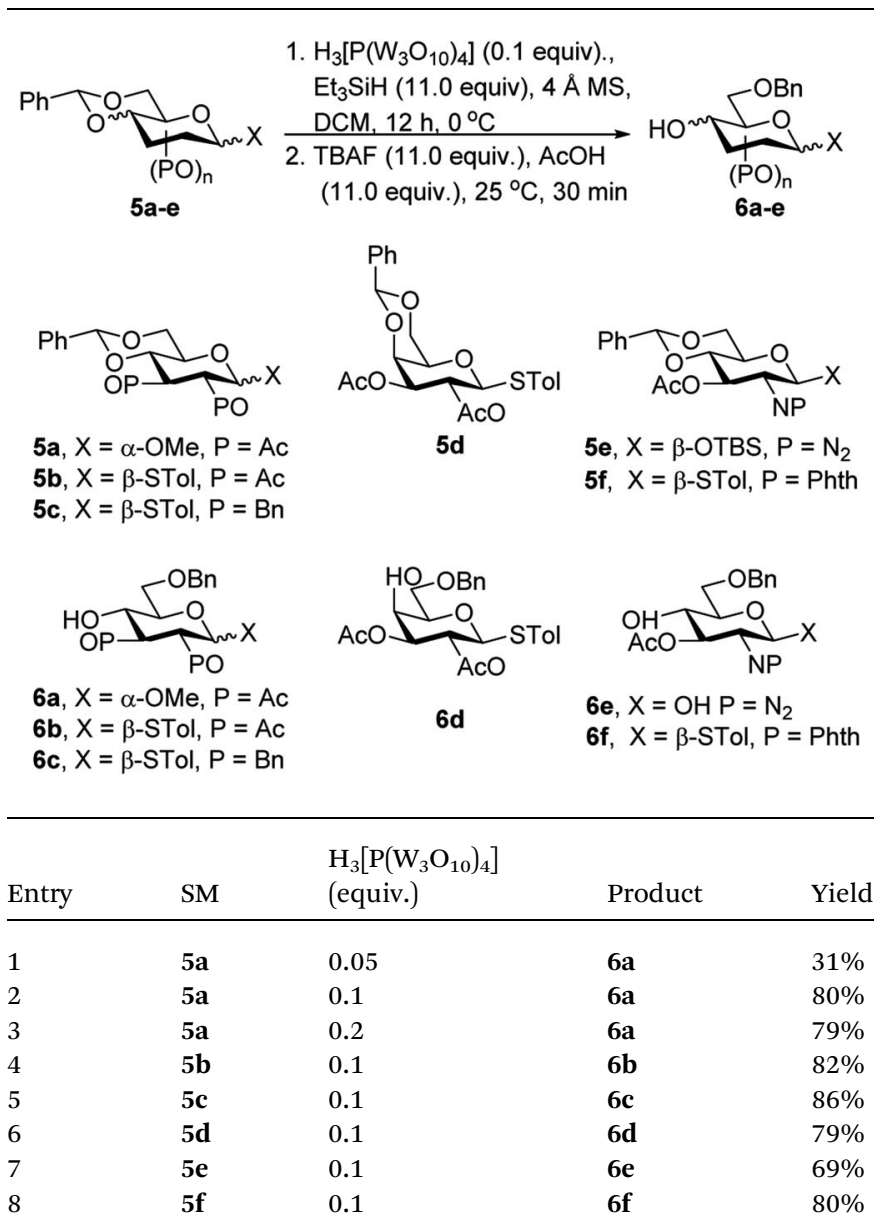


-OTBS was also removed, producing corresponding diol $\mathbf{6 e}$ in $69 \%(\alpha / \beta=3 / 2)$ yield (Table 3, entry 7). Finally, 4-methylphenyl 3-O-acetyl-4,6-O-benzylidene-2-deoxyl-2-phthalimido-1-thio- $\beta$-Dglucopyranoside $5 \mathbf{f}$ freely underwent the reaction, giving the corresponding product 6 f in $80 \%$ yield (Table 3 , entry 8 ). The PTA is little higher cost than TFA. However, PTA brought better yield than TFA ${ }^{12 i}$ catalysed $O-4$ reductive ring-opening reaction.

\section{Glycosylation reactions}

Obtaining highly stereo-selective glycosidic linkages is one of the most challenges in carbohydrate synthesis, as it is affected by solvent effect, neighboring group effect, anomeric effect, temperature effect and promoter effect. Use of phosphotungstic acid which is a large catalyst may lead to increased selectivities in glycosylations. Phosphotungstic acid was used in the glycosylation to synthesize biologically important disaccharide molecules. Chitosan plays a crucial role in tissue repair, angiogenesis, tumor growth and drug delivery. ${ }^{29}$ The disaccharide of chitosan contains $\beta$-D-GlcNH $2(1 \rightarrow 4)-\mathrm{D}-\mathrm{GlcNH}_{2}$ 7. Lipid $A$ is a lipophilic portion of bacterial lipopolysaccharides and has a $\beta$-D-GlcNH ${ }_{2}(1 \rightarrow 6)$-D-GlcNH ${ }_{2}$ backbone $8 .^{30}$ Hyaluronic acid is another important biological molecule, ${ }^{31}$ which is involved in cell-migration, tumor inhibition, adhesion and other processes. It consists of a $\beta$-D-GlcA $(1 \rightarrow 3)$-D-GlcNH $H_{2}$ disaccharide repeating unit 9 (Fig. 2).

During the glycosylation reaction, the acid-sensitive group such as the benzylidene ring was stable and exhibited good tolerance of the phosphotungstic acid catalyst. The catalyst was used as a promoter in the preparation of a disaccharide 13. With donor $\mathbf{5 f}$ and acceptor $\mathbf{6 g}$. The reaction proceeded conveniently to provide compound 13 in $82 \%$ yield (Table 4 , entry 1 ). The derivatives of lipid A have immune-modulator characteristic. They are therefore used as adjuvants for vaccinations and treatments for many diseases. Two protected disaccharides 14 (83\%) and 15 (53\%) were synthesized using phosphotungstic acid (Table 4, entries 2-3). Eventually, phosphotungstic acid was used to synthesize 1,3-linked disaccharide with acceptor 4c and protected donors $5 f$ and 10. The expected products 16 and 17 were obtained in good yields (Table 4, entries 4-5). Three biologically important disaccharide backbones were prepared exclusively in $\beta$-form with the participation of the neighbouring

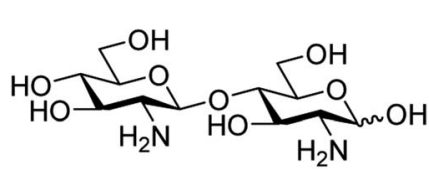

7

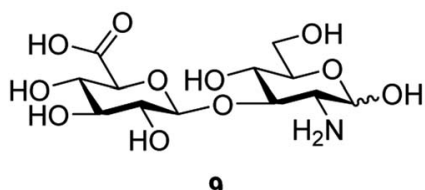

Fig. 2 Structures of the disaccharide of chitosan 7, lipid A 8 and hyaluronic acid 9 .
Table 4 Phosphotungstic acid catalyzed glycosylation with various donors and acceptors

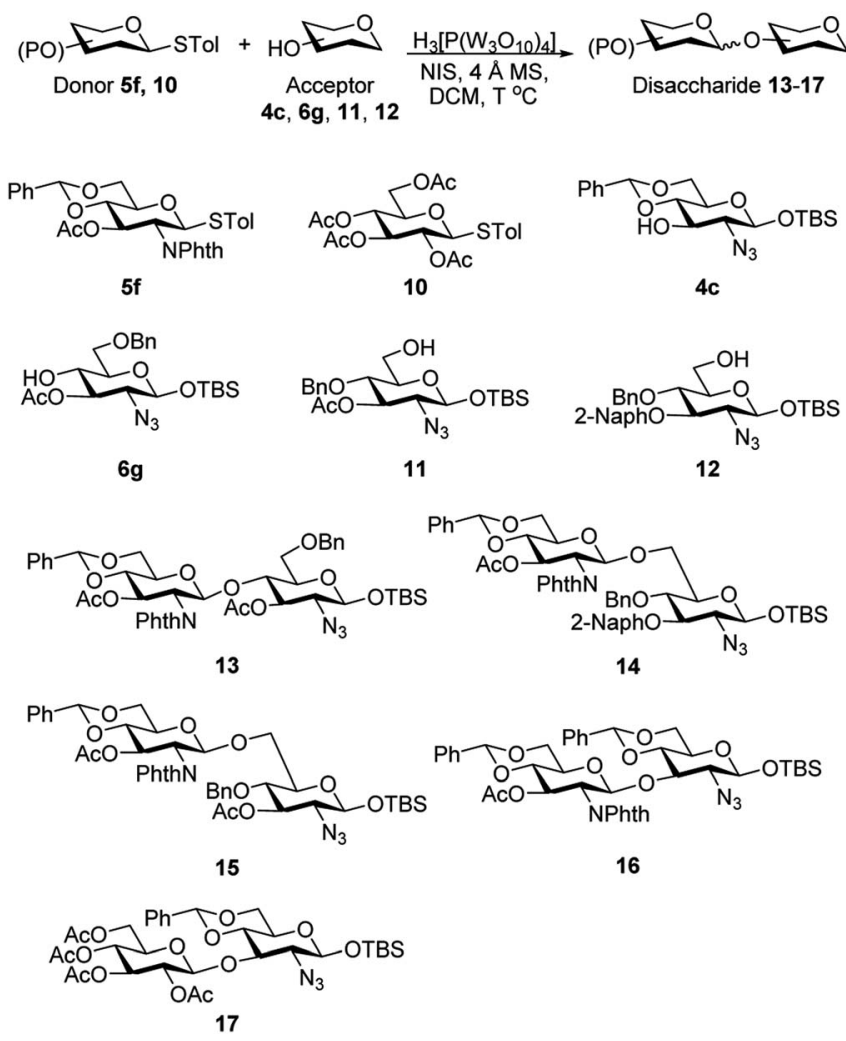

\begin{tabular}{llllll}
\hline Entry & Donor & Acceptor & $T^{\circ} \mathrm{C}$ & Product $(\alpha / \beta)$ & Yield \\
\hline 1 & $\mathbf{5 f}$ & $\mathbf{6 g}$ & 25 & $\mathbf{1 3}(\beta$ only $)$ & $82 \%$ \\
2 & $\mathbf{5 f}$ & $\mathbf{1 1}$ & 0 & $\mathbf{1 4}(\beta$ only $)$ & $83 \%$ \\
3 & $\mathbf{5 f}$ & $\mathbf{1 2}$ & -40 & $\mathbf{1 5}(\beta$ only $)$ & $53 \%$ \\
4 & $\mathbf{5 f}$ & $\mathbf{4 c}$ & 25 & $\mathbf{1 6}(\beta$ only $)$ & $86 \%$ \\
5 & $\mathbf{1 0}$ & $\mathbf{4 c}$ & 25 & $\mathbf{1 7}(\beta$ only $)$ & $67 \%$ \\
\hline
\end{tabular}

group. All of the reactions proceeded smoothly in the presence of phosphotungstic acid as the catalyst. The glycosylation proceeded even at room temperature in the presence of PTA while other catalyst such as $\mathrm{BF}_{3}$.THF, TMSOTf and $\mathrm{Ag}(\mathrm{OTf})_{2}$ required lower temperature.

\section{Conclusions}

We have successfully established that the phosphotungstic acid was a convenient candidate to replace the corresponding homogeneous acids for various carbohydrates reactions. It could effectively catalyze per- $O$-acetylation, 4,6-O-benzylidene acetal formation, regioselective $O-4$ ring-opening, and glycosylation. Notably, the glycosylation reactions brought biologically important disaccharide unites like chitosan, hyaluronic acid and lipid $\mathrm{A}$ in ambient condition. The different protecting groups such as benzylidene acetal, -OAc, -NPhth, -OBn, 2Naph, -OTBS and azido were well tolerated during the reaction. It provides moderate to excellent yields under optimal reaction 
conditions. Phosphotungstic acid is a useful acidic catalyst for various reactions of carbohydrates.

\section{Experimental section}

\section{General information}

The reactions were conducted in flame-dried glassware, under the nitrogen atmosphere. Acetonitrile and dichloromethane were purified and dried from a safe purification system containing activated $\mathrm{Al}_{2} \mathrm{O}_{3}$. All reagents obtained from commercial sources were used without purification unless otherwise mentioned. Flash column chromatography was carried out on Silica Gel 60. TLC was performed on pre-coated glass plates of Silica Gel 60 F254 detection was executed by spraying with a solution of $\mathrm{Ce}\left(\mathrm{NH}_{4}\right)_{2}\left(\mathrm{NO}_{3}\right)_{6}(0.5 \mathrm{~g}),\left(\mathrm{NH}_{4}\right)_{6} \mathrm{Mo}_{7} \mathrm{O}_{24}(24.0 \mathrm{~g})$ and $\mathrm{H}_{2} \mathrm{SO}_{4}(28.0 \mathrm{~mL})$ in water $(500.0 \mathrm{~mL})$ and subsequent heating on a hot plate. Optical rotations were measured at $589 \mathrm{~nm}(\mathrm{Na}),{ }^{1} \mathrm{H}$, ${ }^{13} \mathrm{C}$ NMR, DEPT, ${ }^{1} \mathrm{H}^{-1} \mathrm{H}$ COSY, ${ }^{1} \mathrm{H}_{-}{ }^{13} \mathrm{C}$ COSY, and NOESY spectra were recorded with $400 \mathrm{MHz}$ instruments. Chemical shifts are in ppm from $\mathrm{Me}_{4} \mathrm{Si}$ generated from the $\mathrm{CDCl}_{3}$ lock signal at $\delta 7.26$. IR spectra were taken with a FT-IR spectrometer using $\mathrm{NaCl}$ plates. Mass spectra were analyzed on orbitrap instrument with an ESI source.

\section{Acetylation}

General procedure for per- $\mathrm{O}$-acetylation reaction. A round bottom flask equipped with a magnetic stirrer bar was charged with 1a-1f (200 $\mathrm{mg}$ for 1a-1e, $500 \mathrm{mg}$ for 1f, 1.0 equiv.) and to this were added phosphotungstic acid ( 0.02 equiv.) and acetic anhydride (10.0 equiv.) under nitrogen atmosphere. The reaction mixture was stirred at $28{ }^{\circ} \mathrm{C}$ for 24 hours. After completion of the reaction, the reaction mixture was diluted with ethyl acetate and extracted with water $(50.0 \mathrm{~mL} \times 3)$. The combined organic layers were dried over anhydrous $\mathrm{MgSO}_{4}$, filtered and concentrated. The crude product was purified by column chromatography on silica gel to give the desired product $2 \mathbf{a}-\mathbf{2 f}$.

1,2,3,4,6-Penta-O-acetyl-D-glucopyranose (2a). Prepared according to the general procedure discussed above: white solid (405 mg, 93\%); $R_{\mathrm{f}} 0.53$ (EtOAc/Hex = 1/1); mp 109-113 ${ }^{\circ} \mathrm{C}$; $[\alpha]_{\mathrm{D}}^{29}+62.5(c 1.0, \mathrm{DCM})$; IR (NaCl) $\nu 1752,1647,1371,1221$, $1147 \mathrm{~cm}^{-1} ;{ }^{1} \mathrm{H}$ NMR $\left(400 \mathrm{MHz}, \mathrm{CDCl}_{3}\right) \delta 6.33(\mathrm{~d}, J=3.6 \mathrm{~Hz}, 1 \mathrm{H})$, $5.71(\mathrm{~d}, J=8.4 \mathrm{~Hz}, 0.2 \mathrm{H}), 5.50-5.45(\mathrm{~m}, 1 \mathrm{H}), 5.25(\mathrm{t}, J=9.2 \mathrm{~Hz}$, $0.2 \mathrm{H}), 5.16-5.14(\mathrm{~m}, 1 \mathrm{H}), 5.11(\mathrm{~d}, J=4.0 \mathrm{~Hz}, 1 \mathrm{H}), 5.09(\mathrm{~d}, J=$ $4.0 \mathrm{~Hz}, 0.5 \mathrm{H}), 4.29-4.28(\mathrm{~m}, 0.5 \mathrm{H}), 4.25(\mathrm{~d}, J=4.4 \mathrm{~Hz}, 1 \mathrm{H}), 4.14-$ $4.10(\mathrm{~m}, 2 \mathrm{H}), 4.07(\mathrm{~d}, J=2.4 \mathrm{~Hz}, 0.4 \mathrm{H}), 2.18(\mathrm{~s}, 3 \mathrm{H}), 2.12(\mathrm{~s}$, $0.6 \mathrm{H}), 2.10(\mathrm{~s}, 3 \mathrm{H}), 2.09(\mathrm{~s}, 0.7 \mathrm{H}), 2.04(\mathrm{~s}, 3 \mathrm{H}), 2.04(\mathrm{~s}, 0.5 \mathrm{H}), 2.03$ (s, 0.5H), $2.03(\mathrm{~s}, 3 \mathrm{H}), 2.02(\mathrm{~s}, 3 \mathrm{H}), 2.01(\mathrm{~s}, 0.6 \mathrm{H}) ;{ }^{13} \mathrm{C}$ NMR $(100$ $\left.\mathrm{MHz}, \mathrm{CDCl}_{3}\right) \delta 170.6,170.2,169.6,169.4,168.7,89.0,69.8,69.2$, 67.9, 61.4, 20.9, 20.7, 20.6, 20.5, 20.4; HRMS (ESI, M + Na ${ }^{+}$) calcd for $\mathrm{C}_{16} \mathrm{H}_{22} \mathrm{O}_{11} \mathrm{Na}$ 413.1060, found 413.1050.

1,2,3,4,6-Penta-O-acetyl-D-galactopyranose (2b). Prepared according to the general procedure discussed above: white soild (353 mg, 81\%); $R_{\mathrm{f}} 0.50$ (EtOAc/Hex $=1 / 2$ ); mp 70-80 ${ }^{\circ} \mathrm{C}$; $[\alpha]_{\mathrm{D}}^{29}+80.8(c 1.0, \mathrm{DCM}) ; \mathrm{IR}(\mathrm{NaCl}) \nu 1751,1648,1373 \mathrm{~cm}^{-1} ;{ }^{1} \mathrm{H}$ NMR $\left(400 \mathrm{MHz}, \mathrm{CDCl}_{3}\right) \delta 6.38(\mathrm{~d}, J=1.6 \mathrm{~Hz}, 1 \mathrm{H}), 5.69(\mathrm{~d}, J=$ $8.4 \mathrm{~Hz}, 0.2 \mathrm{H}), 5.50(\mathrm{~d}, J=1.2 \mathrm{~Hz}, 1 \mathrm{H}), 5.42(\mathrm{dd}, J=3.2,1.0 \mathrm{~Hz}$,
0.2H), $5.33(\mathrm{t}, J=1.4 \mathrm{~Hz}, 2 \mathrm{H}), 5.09-5.05(\mathrm{~m}, 0.2 \mathrm{H}), 4.34(\mathrm{td}, J=$ $6.8,1.2 \mathrm{~Hz}, 1 \mathrm{H}), 4.14(\mathrm{t}, J=7.0 \mathrm{~Hz}, 0.4 \mathrm{H}), 4.10(\mathrm{~d}, J=3.3 \mathrm{~Hz}, 1 \mathrm{H})$, $4.09(\mathrm{~d}, J=3.4 \mathrm{~Hz}, 1 \mathrm{H}), 4.07-4.05(\mathrm{~m}, 0.3 \mathrm{H}), 2.17(\mathrm{~m}, 0.6 \mathrm{H}), 2.16$ (s, 3H), $2.16(\mathrm{~s}, 3 \mathrm{H}), 2.12(\mathrm{~s}, 0.6 \mathrm{H}), 2.05(\mathrm{~s}, 0.5 \mathrm{H}), 2.04(\mathrm{~s}, 3 \mathrm{H})$, $2.02(\mathrm{~s}, 3 \mathrm{H}), 2.01(\mathrm{~s}, 3 \mathrm{H}), 1.99(\mathrm{~s}, 0.5 \mathrm{H}) ;{ }^{13} \mathrm{C}$ NMR $(100 \mathrm{MHz}$, $\left.\mathrm{CDCl}_{3}\right) \delta 170.0,169.9,169.8,169.6,168.6,89.4,68.5,67.2,67.1$, 66.2, 61.0, 20.6, 20.5, 20.4, 20.3; HRMS (ESI, $\mathrm{M}+\mathrm{Na}^{+}$) calcd for $\mathrm{C}_{16} \mathrm{H}_{22} \mathrm{O}_{11} \mathrm{Na}$ 413.1060, found 413.1056.

1,2,3,4,6-Penta-O-acetyl- $\alpha$-D-mannopyranose (2c). Prepared according to the general procedure discussed above: colorless oil $(380 \mathrm{mg}, 88 \%) ; R_{\mathrm{f}} 0.60(\mathrm{EtOAc} / \mathrm{Hex}=1 / 1) ;[\alpha]_{\mathrm{D}}^{29}+50.3(c 1.0$, DCM); IR (NaCl) $\nu 2106,1749,1645,1371 \mathrm{~cm}^{-1} ;{ }^{1} \mathrm{H}$ NMR (400 $\left.\mathrm{MHz}, \mathrm{CDCl}_{3}\right) \delta 6.08(\mathrm{~s}, 1 \mathrm{H}), 5.34-5.33(\mathrm{~m}, 2 \mathrm{H}), 5.25(\mathrm{~s}, 1 \mathrm{H}), 4.28$ $(\mathrm{dd}, J=12.0,4.8 \mathrm{~Hz}, 1 \mathrm{H}), 4.08(\mathrm{dd}, J=12.6,1.8 \mathrm{~Hz}, 2 \mathrm{H}), 2.17(\mathrm{~s}$, $3 \mathrm{H}), 2.16(\mathrm{~s}, 3 \mathrm{H}), 2.09(\mathrm{~s}, 3 \mathrm{H}), 2.05(\mathrm{~s}, 3 \mathrm{H}), 2.00(\mathrm{~s}, 3 \mathrm{H}) ;{ }^{13} \mathrm{C} \mathrm{NMR}$ $\left(100 \mathrm{MHz}, \mathrm{CDCl}_{3}\right) \delta 170.3,169.7,169.4,169.3,167.8,90.3,70.3$, $68.5,68.0,65.2,61.8,20.5,20.4,20.3,20.29,20.26$; HRMS (ESI, $\mathrm{M}+\mathrm{Na}^{+}$) calcd for $\mathrm{C}_{16} \mathrm{H}_{22} \mathrm{O}_{11} \mathrm{Na} 413.1060$, found 413.1054.

Methyl 2,3,4,6-tetra- $O$-acetyl- $\alpha$-D-glucopyranoside (2d). Prepared according to the general procedure discussed above: white soild (330 mg, 88\%); $R_{\mathrm{f}} 0.60$ (EtOAc/Hex $\left.=1 / 2\right)$; mp 51$53{ }^{\circ} \mathrm{C} ;[\alpha]_{\mathrm{D}}^{29}+46.7$ (c 1.0, DCM); IR (NaCl) $\nu 2960,1750,1648$, $1371,1225 \mathrm{~cm}^{-1} ;{ }^{1} \mathrm{H} \mathrm{NMR}\left(400 \mathrm{MHz}, \mathrm{CDCl}_{3}\right) \delta 5.47(\mathrm{dd}, J=10.1$, $9.5 \mathrm{~Hz}, 1 \mathrm{H}), 5.07$ (dd, $J=10.2,9.4 \mathrm{~Hz}, 1 \mathrm{H}), 4.95(\mathrm{~d}, J=3.7 \mathrm{~Hz}$, 1H), $4.90(\mathrm{dd}, J=10.2,3.7 \mathrm{~Hz}, 1 \mathrm{H}), 4.26(\mathrm{dd}, J=12.3,4.6 \mathrm{~Hz}$, 1H), 4.12-4.08 (m, 1H), 3.98 (ddd, $J=10.2,4.5,2.3 \mathrm{~Hz}, 1 \mathrm{H}), 3.41$ (s, 3H), $2.10(\mathrm{~s}, 3 \mathrm{H}), 2.08(\mathrm{~s}, 3 \mathrm{H}), 2.03(\mathrm{~s}, 3 \mathrm{H}), 2.01(\mathrm{~s}, 3 \mathrm{H}) ;{ }^{13} \mathrm{C}$ NMR $\left(100 \mathrm{MHz}, \mathrm{CDCl}_{3}\right) \delta$ 170.4, 169.9, 169.8, 169.4, 96.6, 70.6, 69.9, 68.3, 67.0, 61.7, 55.3, 20.5, 20.46, 20.4; HRMS (ESI, M + $\mathrm{Na}^{+}$) calcd for $\mathrm{C}_{15} \mathrm{H}_{22} \mathrm{O}_{10} \mathrm{Na}$ 385.1111, found 385.1111.

1,2,3,5-Tetra-O-acetate-D-xylopyranose (2e). Prepared according to the general procedure discussed above: colorless oil (424 mg, 99\%); $R_{\mathrm{f}} 0.55(\mathrm{EtOAc} / \mathrm{Hex}=1 / 1) ;[\alpha]_{\mathrm{D}}^{29}+51.8(c 1.0$, DCM); IR ( NaCl) $\nu 2924,1743,1649,1370,1211 \mathrm{~cm}^{-1} ;{ }^{1} \mathrm{H}$ NMR $\left(400 \mathrm{MHz}, \mathrm{CDCl}_{3}\right) \delta 6.25(\mathrm{~d}, J=3.6 \mathrm{~Hz}, 1 \mathrm{H}), 5.70(\mathrm{~d}, J=6.8 \mathrm{~Hz}$, $0.18 \mathrm{H}), 5.46(\mathrm{t}, J=9.9 \mathrm{~Hz}, 1 \mathrm{H}), 5.36(\mathrm{t}, J=5.0 \mathrm{~Hz}, 0.33 \mathrm{H}), 5.30-$ $5.23(\mathrm{~m}, 0.28 \mathrm{H}), 5.19(\mathrm{t}, J=8.4 \mathrm{~Hz}, 0.26 \mathrm{H}), 5.06-5.00(\mathrm{~m}, 2 \mathrm{H})$, $4.35(\mathrm{dd}, J=12.0,4.0 \mathrm{~Hz}, 0.22 \mathrm{H}), 4.24(\mathrm{~d}, J=6.0 \mathrm{~Hz}, 0.15 \mathrm{H}), 4.14$ $(\mathrm{dd}, J=12.4,4.8 \mathrm{~Hz}, 0.24 \mathrm{H}), 3.99(\mathrm{dd}, J=12.0,6.0 \mathrm{~Hz}, 0.23 \mathrm{H})$, $3.93(\mathrm{dd}, J=11.2,5.9 \mathrm{~Hz}, 1 \mathrm{H}), 3.70(\mathrm{t}, J=11.0 \mathrm{~Hz}, 1 \mathrm{H}), 3.52(\mathrm{dd}$, $J=12.0,8.4 \mathrm{~Hz}, 0.2 \mathrm{H}) .2 .17(\mathrm{~s}, 3 \mathrm{H}), 2.13-2.05(\mathrm{~m}, 9 \mathrm{H}), 2.05(\mathrm{~s}$, $3 \mathrm{H}), 2.04(\mathrm{~s}, 3 \mathrm{H}), 2.02(\mathrm{~s}, 3 \mathrm{H}) ;{ }^{13} \mathrm{C}$ NMR $\left(100 \mathrm{MHz}, \mathrm{CDCl}_{3}\right)$ $\delta 170.0,169.7,169.6,168.9,89.1,69.2,69.16,68.5,60.5,20.8$, 20.6, 20.6, 20.4; HRMS (ESI, $\mathrm{M}+\mathrm{Na}^{+}$) calcd for $\mathrm{C}_{13} \mathrm{H}_{18} \mathrm{O}_{9} \mathrm{Na}$ 341.0849 , found 341.0842 .

1,2,3,6-Tetra- $O$-acetate-4-O-(2,3,4,6-tetra- $O$-acetyl- $\beta$-D-galactopyranosyl)-D-glucopyranose (2f). Prepared according to the general procedure discussed above: white solid (950 mg, 96\%); $R_{\mathrm{f}} 0.44(\mathrm{EtOAc} / \mathrm{Hex}=3 / 2) ; \mathrm{mp} 85-87^{\circ} \mathrm{C} ;[\alpha]_{\mathrm{D}}^{22}+55.8(c 1.0, \mathrm{DCM}) ;$ IR (NaCl) $\nu$ 2981, 2943, 1753, 1649, 1434, $1371 \mathrm{~cm}^{-1} ;{ }^{1} \mathrm{H}$ NMR $\left(400 \mathrm{MHz}, \mathrm{CDCl}_{3}\right) \delta 6.25(\mathrm{~d}, J=3.6 \mathrm{~Hz}, 1 \mathrm{H}), 5.67(\mathrm{~d}, J=8.4 \mathrm{~Hz}$, $0.2 \mathrm{H}), 5.46(\mathrm{t}, J=9.6 \mathrm{~Hz}, 1.2 \mathrm{H}), 5.35(\mathrm{~d}, J=3.6 \mathrm{~Hz}, 1.2 \mathrm{H}), 5.27-$ $5.21(\mathrm{~m}, 0.3 \mathrm{H}), 5.12(\mathrm{dd}, J=10.4,7.6 \mathrm{~Hz}, 1 \mathrm{H}), 5.09-5.03(\mathrm{~m}$, $0.4 \mathrm{H}), 5.00(\mathrm{dd}, J=10.3,3.6 \mathrm{~Hz}, 1 \mathrm{H}), 4.96(\mathrm{dd}, J=10.2,3.4 \mathrm{~Hz}$, $1 \mathrm{H}), 4.48(\mathrm{~d}, J=8.0 \mathrm{~Hz}, 1 \mathrm{H}), 4.43(\mathrm{~s}, 1 \mathrm{H}), 4.18-4.05(\mathrm{~m}, 4 \mathrm{H}), 4.00$ (ddd, $J=10.0,3.6,1.6 \mathrm{~Hz}, 1 \mathrm{H}), 3.88(\mathrm{t}, J=6.8 \mathrm{~Hz}, 1 \mathrm{H}), 3.81(\mathrm{t}, J=$ $9.7 \mathrm{~Hz}, 1 \mathrm{H}), 2.18(\mathrm{~s}, 3 \mathrm{H}), 2.16(\mathrm{~s}, 2 \mathrm{H}), 2.15(\mathrm{~s}, 1 \mathrm{H}), 2.13(\mathrm{~s}, 3 \mathrm{H})$, 
$2.12(\mathrm{~s}, 1 \mathrm{H}), 2.09(\mathrm{~s}, 1 \mathrm{H}), 2.06(\mathrm{~s}, 2 \mathrm{H}), 2.06(\mathrm{~s}, 2 \mathrm{H}), 2.05(\mathrm{~s}, 3 \mathrm{H})$, 2.05 (s, 1H), 2.04 (s, 1H), $2.03(\mathrm{~s}, 1 \mathrm{H}), 2.01$ (s, 2H), 1.97 (s, 3H); ${ }^{13} \mathrm{C} \mathrm{NMR}\left(100 \mathrm{MHz}, \mathrm{CDCl}_{3}\right) \delta 170.28,170.24,170.08,170.01$, $169.87,169.56,169.07,168.88,101.13,88.87,75.72,70.91$, $70.61,69.50,69.31,69.01,66.49,61.36,60.70,20.91,20.80$, 20.61, 20.46; HRMS (ESI, $\mathrm{M}+\mathrm{Na}^{+}$) calcd for $\mathrm{C}_{28} \mathrm{H}_{38} \mathrm{O}_{19} \mathrm{Na}$ 701.1905, found 701.1902.

\section{Acetalization}

General procedure for benzylidene formation. A solution of 1d, 3b-3e (150 mg for 1d, 3b, 3d, and 3e, $140 \mathrm{mg}$ for 3c, and $120 \mathrm{mg}$ for $3 \mathrm{e}, 1.0$ equiv.) and activated $4 \AA \mathrm{AS}$ in anhydrous acetonitrile $(2 \mathrm{~mL})$ was stirred for 30 minutes under nitrogen atmosphere at rt. Then the flask was placed into an ice bath. After 3 minutes, benzaldehyde dimethyl acetal (2.0 equiv.) and dried phosphotungstic acid ( 0.3 equiv. for $\mathbf{1 d}, \mathbf{3 b}, \mathbf{3 d}$, and $\mathbf{3 e}$ and 0.15 equiv. for $3 \mathbf{c}$ ) were added to the reaction mixture at $0{ }^{\circ} \mathrm{C}$. After completion of the reaction, the reaction mixture was passed through the Celite and extracted with water $(25 \mathrm{~mL})$ and ethyl acetate $(50 \mathrm{~mL} \times 2)$. The combined organic layers were washed with brine, dried over anhydrous $\mathrm{MgSO}_{4}$, filtered and concentrated under reduced pressure. The crude product was purified by column chromatography on silica gel to afford $\mathbf{4 a -}$ 4e.

Methyl 4,6-O-benzylidene- $\alpha$-D-glucopyranoside

(4a). Prepared according to the general procedure discussed above: white solid. (157 mg, 72\%); $R_{\mathrm{f}} 0.35$ (EtOAc); mp 161-162 ${ }^{\circ} \mathrm{C}$; $[\alpha]_{\mathrm{D}}^{25}+117.0$ (c 1.0, DCM); IR (NaCl) v 3380, 2915, 2870, 1640, 1453, 1373, 1192, 1145, 1125, 1076, 1030, $1000 \mathrm{~cm}^{-1} ;{ }^{1} \mathrm{H}$ NMR $\left(400 \mathrm{MHz}, \mathrm{CDCl}_{3}\right) \delta 7.48-7.45(\mathrm{~m}, 2 \mathrm{H}), 7.37-7.32(\mathrm{~m}, 3 \mathrm{H}), 5.49$ $(\mathrm{s}, 1 \mathrm{H}), 4.73(\mathrm{~d}, J=4.0 \mathrm{~Hz}, 1 \mathrm{H}), 4.26(\mathrm{dd}, J=9.6,4.4 \mathrm{~Hz}, 1 \mathrm{H})$, $3.88(\mathrm{t}, J=9.2 \mathrm{~Hz}, 1 \mathrm{H}), 3.80-3.67(\mathrm{~m}, 2 \mathrm{H}), 3.58(\mathrm{bs}, 1 \mathrm{H}), 3.44(\mathrm{t}, J$ $=12.0 \mathrm{~Hz}, 1 \mathrm{H}), 3.41(\mathrm{~s}, 3 \mathrm{H}), 3.29(\mathrm{bs}, 1 \mathrm{H}), 2.65(\mathrm{bs}, 1 \mathrm{H}) ;{ }^{13} \mathrm{C}$ NMR (100 MHz, CDCl3) $\delta 137.2,129.5,128.6,126.5,102.1$, 100.0, 81.1, 73.0, 71.8, 69.1, 62.8, 55.8; HRMS (ESI, $\mathrm{M}+\mathrm{Na}^{+}$) calcd for $\mathrm{C}_{14} \mathrm{H}_{18} \mathrm{O}_{6} \mathrm{Na} 305.1001$, found 305.1005.

4-Methylphenyl 4,6-O-benzylidene-2-deoxyl-1-thio-2-phthalimido- $\boldsymbol{\beta}$-D-glucopyranoside (4b). Prepared according to the general procedure discussed above: white solid (154 mg, 84\%); $R_{\mathrm{f}} 0.46($ EtOAc/Hex $=1 / 2) ; \operatorname{mp} 121-122{ }^{\circ} \mathrm{C} ;[\alpha]_{\mathrm{D}}^{25}+40.0(c \quad 0.45$, $\mathrm{DCM})$; IR $(\mathrm{NaCl}) v 3443,2868,2090,1775,1644 \mathrm{~cm}^{-1} ;{ }^{1} \mathrm{H}$ NMR $\left(400 \mathrm{MHz}, \mathrm{CDCl}_{3}\right) \delta$ 7.91-7.78 (m, 2H), 7.76-7.68 (m, 2H), 7.49$4.41(\mathrm{~m}, 2 \mathrm{H}), 7.38-7.31(\mathrm{~m}, 3 \mathrm{H}), 7.27-7.24(\mathrm{~m}, 2 \mathrm{H}), 7.05(\mathrm{~d}, J=$ $7.6 \mathrm{~Hz}, 2 \mathrm{H}), 5.60(\mathrm{~d}, J=10.8 \mathrm{~Hz}, 1 \mathrm{H}), 5.53(\mathrm{~s}, 1 \mathrm{H}), 4.58(\mathrm{t}, J=$ $8.8 \mathrm{~Hz}, 1 \mathrm{H}), 4.36$ (dd, $J=10.4,4.8 \mathrm{~Hz}, 1 \mathrm{H}), 4.27(\mathrm{t}, J=10.0 \mathrm{~Hz}$, $1 \mathrm{H}), 3.79(\mathrm{t}, J=10.0 \mathrm{~Hz}, 1 \mathrm{H}), 3.68-3.60(\mathrm{~m}, 1 \mathrm{H}), 3.55(\mathrm{t}, J=$ $9.2 \mathrm{~Hz}, 1 \mathrm{H}), 2.75(\mathrm{~s}, 1 \mathrm{H}), 2.29(\mathrm{~s}, 3 \mathrm{H}) ;{ }^{13} \mathrm{C} \mathrm{NMR}\left(100 \mathrm{MHz}, \mathrm{CDCl}_{3}\right)$ $\delta$ 165.7, 165.0, 135.9, 134.3, 131.6, 130.7, 129.1, 127.1, 126.8, 125.8, 125.2, 123.7, 121.3, 120.8, 99.3, 81.9, 79.3, 67.7, 67.1, 66.0, 53.0, 18.6; HRMS (ESI, $\mathrm{M}+\mathrm{Na}^{+}$) calcd for $\mathrm{C}_{28} \mathrm{H}_{25} \mathrm{NO}_{6} \mathrm{SNa}$ 526.1301 , found 526.1299 .

2-Azido-4,6-O-benzylidene-2-deoxyl-1-O-tert-butyldi-methylsilyl- $\beta$-D-glucopyranoside (4c). Prepared according to the general procedure discussed above: colorless oil. (106 mg, 82\%); $R_{\mathrm{f}} 0.59(\mathrm{EtOAc} / \mathrm{Hex}=1 / 2) ;[\alpha]_{\mathrm{D}}^{25}-19.9(c 0.05, \mathrm{DCM}) ; \mathrm{IR}(\mathrm{NaCl}) v$ 3443, 3039, 2860, $2111 \mathrm{~cm}^{-1} ;{ }^{1} \mathrm{H}$ NMR (400 MHz, $\left.\mathrm{CDCl}_{3}\right) \delta 7.52-$ $7.47(\mathrm{~m}, 2 \mathrm{H}), 7.41-7.35(\mathrm{~m}, 3 \mathrm{H}), 5.50(\mathrm{~s}, 1 \mathrm{H}), 4.59$ (d, J=8.0 Hz, $1 \mathrm{H}), 4.26(\mathrm{t}, J=4.0 \mathrm{~Hz}, 1 \mathrm{H}), 3.74(\mathrm{t}, J=10.0 \mathrm{~Hz}, 1 \mathrm{H}), 3.59-3.46$ (m, 2H), 3.31-3.25 (m, 2H), $3.18(\mathrm{br}, 1 \mathrm{H}), 0.97(\mathrm{~s}, 9 \mathrm{H}), 0.18$ (s, $6 \mathrm{H}) ;{ }^{13} \mathrm{C}$ NMR $\left(100 \mathrm{MHz}, \mathrm{CDCl}_{3}\right) \delta 136.8,129.2,128.3,126.3$, 101.8, 97.4, 80.7, 71.5, 68.9, 68.4, 66.2, 25.5, 17.8, -4.4, -5.3; HRMS (ESI, $\mathrm{M}+\mathrm{Na}^{+}$) calcd for $\mathrm{C}_{19} \mathrm{H}_{29} \mathrm{~N}_{3} \mathrm{O}_{5} \mathrm{SiNa} 430.1774$, found 430.1770 .

4-Methylphenyl 4,6-O-benzylidene-1-thio- $\beta$-D-glucopyranoside (4d). Prepared according to the general procedure discussed above: white solid. (126 mg, 65\%); $R_{\mathrm{f}} 0.62$ (EtOAc); mp $177-178{ }^{\circ} \mathrm{C}$; $[\alpha]_{\mathrm{D}}^{25}-161.30$ (c 1.0, DCM); IR (NaCl) v 3442, 2870, 2088, 1643, 1493, 1454, 1381, 1265, 1214, 1165, 1085, 1030, $1005,974 \mathrm{~cm}^{-1} ;{ }^{1} \mathrm{H} \mathrm{NMR}\left(400 \mathrm{MHz}, \mathrm{CDCl}_{3}\right) \delta 7.48-7.42(\mathrm{~m}, 4 \mathrm{H})$, 7.38-7.35 (m, 3H), 7.15 (d, $J=8.0 \mathrm{~Hz}, 2 \mathrm{H}), 5.52(\mathrm{~s}, 1 \mathrm{H}), 4.57$ (d, $J$ $=9.6 \mathrm{~Hz}, 1 \mathrm{H}), 4.39(\mathrm{dd}, J=10.4,4.4 \mathrm{~Hz}, 1 \mathrm{H}), 3.85(\mathrm{t}, J=8.4 \mathrm{~Hz}$, $1 \mathrm{H}), 3.78(\mathrm{t}, J=7.4 \mathrm{~Hz}, 1 \mathrm{H}), 3.52-3.47(\mathrm{~m}, 2 \mathrm{H}), 3.43(\mathrm{t}, J=9.0 \mathrm{~Hz}$, 1H), 2.80 (bs, 1H), 2.65 (bs, $1 \mathrm{H}), 2.36(\mathrm{~s}, 3 \mathrm{H}) ;{ }^{13} \mathrm{C} \mathrm{NMR}(100 \mathrm{MHz}$, $\left.\mathrm{CDCl}_{3}\right) \delta 138.6,136.8,133.4,129.7,129.2,128.2,127.3,126.2$, 101.7, 88.5, 80.0, 74.3, 72.3, 70.3, 68.4, 21.1; HRMS (ESI, M + $\mathrm{Na}^{+}$) calcd for $\mathrm{C}_{20} \mathrm{H}_{22} \mathrm{O}_{5} \mathrm{SNa} 397.1086$, found 397.1084.

4-Methylphenyl 4,6-O-benzylidene-1-thio- $\beta$-D-galactopyranoside (4e). Prepared according to the general procedure discussed above: white solid (135 $\mathrm{mg}, 86 \%) ; R_{\mathrm{f}} 0.34$ (EtOAc); mp $145-146{ }^{\circ} \mathrm{C} ;[\alpha]_{\mathrm{D}}^{25}-175.30$ (c 1.0, DCM); IR (NaCl) v 3440, 2920, 2665, 2248, 1644, 1493, 1451, 1402, 1361, 1276, 1245, 1197, 1165, 1101, 1070, 1042, 993, $970 \mathrm{~cm}^{-1}$; ${ }^{1} \mathrm{H}$ NMR $(400 \mathrm{MHz}$, $\left.\mathrm{CDCl}_{3}\right) \delta 7.45(\mathrm{~d}, J=8.0 \mathrm{~Hz}, 2 \mathrm{H}), 7.25(\mathrm{~d}, J=4.0 \mathrm{~Hz}, 5 \mathrm{H}), 6.99(\mathrm{~d}$, $J=8.0 \mathrm{~Hz}, 2 \mathrm{H}), 5.37(\mathrm{~s}, 1 \mathrm{H}), 4.32(\mathrm{~d}, J=8.8 \mathrm{~Hz}, 1 \mathrm{H}), 4.25(\mathrm{~d}, J=$ $12.4 \mathrm{~Hz}, 1 \mathrm{H}), 4.06$ (d, $J=3.2 \mathrm{~Hz}, 1 \mathrm{H}), 3.89$ (d, $J=12.4 \mathrm{~Hz}, 1 \mathrm{H})$, 3.55 (bs, 1H), 3.50 (t, $J=9.2 \mathrm{~Hz}, 1 \mathrm{H}), 3.40$ (s, 1H), 2.54 (bs, 2H), $2.24(\mathrm{~s}, 3 \mathrm{H}) ;{ }^{13} \mathrm{C}$ NMR $\left(100 \mathrm{MHz}, \mathrm{CDCl}_{3}\right) \delta 138.5,137.5,134.2$, 129.6, 129.2, 128.1, 126.5, 101.3, 86.9, 75.2, 73.7, 69.9, 69.2, 68.6, 21.2; HRMS (ESI, $\mathrm{M}+\mathrm{Na}^{+}$) calcd for $\mathrm{C}_{20} \mathrm{H}_{22} \mathrm{O}_{5} \mathrm{SNa} 397.1086$, found 397.1086 .

\section{Regioselective reductive ring opening of benzylidene acetals}

General procedure for regio-selective $O$-4 ring opening reaction. A solution of $\mathbf{5 a - 5 f}(100 \mathrm{mg}$ for $\mathbf{5 a}-\mathbf{5 e}$ and $200 \mathrm{mg}$ for 5f, 1.0 equiv.) and activated $4 \AA \mathrm{MS}(100 \mathrm{mg})$ in anhydrous dichloromethane $(1.0 \mathrm{~mL})$ was stirred under nitrogen atmosphere at room temperature. Then the flask was placed into an ice bath for 5 minutes and triethylsilane (11 equiv.), phosphotungstic acid ( 0.1 equiv.) were added to the reaction mixture at $0{ }^{\circ} \mathrm{C}$. Then the reaction was allowed to stir at same temperature for 12 hours. After completion of the reaction, were added $1 \mathrm{M}$ tetra- $n$-butyl ammonium fluoride ( 11 equiv.) and acetic acid (11 equiv.) at $0{ }^{\circ} \mathrm{C}$, and stirred for 30 minutes at $25{ }^{\circ} \mathrm{C}$. The molecular sieves were removed by filtration through Celite. The filtrate was extracted with EtOAc $(20 \mathrm{~mL} \times 3)$ and water $(20 \mathrm{~mL})$. The combined organic layers were dried over anhydrous $\mathrm{MgSO}_{4}$, filtered and concentrated. It was purified by column chromatography on silica gel to give the desired product $\mathbf{6 a - 6 f}$.

Methyl 2,3-di-O-acetyl-6-O-benzyl- $\alpha$-D-glucopyranoside (6a). Prepared according to the general procedure discussed above: yellow liquid (88 mg, 80\%); $R_{\mathrm{f}} 0.35$ (EtOAc/Hex $=1 / 1$ ); $[\alpha]_{\mathrm{D}}^{26}+109.5$ (c 1.0, DCM); IR (NaCl) $\nu$ 3474, 2919, 2871, 1747, 
1452, $1371 \mathrm{~cm}^{-1} ;{ }^{1} \mathrm{H}$ NMR (400 MHz, $\left.\mathrm{CDCl}_{3}\right) \delta 7.37-7.29(\mathrm{~m}$, $5 \mathrm{H}), 5.30(\mathrm{t}, J=10.0 \mathrm{~Hz} 1 \mathrm{H}), 4.91(\mathrm{~d}, J=3.6 \mathrm{~Hz}, 1 \mathrm{H}), 4.87(\mathrm{dd}, J=$ $10.1,3.6 \mathrm{~Hz}, 1 \mathrm{H}), 4.60$ (q, $J=12.0 \mathrm{~Hz}, 2 \mathrm{H}), 3.80-3.70(\mathrm{~m}, 4 \mathrm{H})$, 3.40 (s, 3H), 2.83 (s, 1H), 2.09 (s, 3H), 2.08 (s, 3H).; ${ }^{13} \mathrm{C}$ NMR $(100$ $\left.\mathrm{MHz}, \mathrm{CDCl}_{3}\right) \delta 176.8,171.3,170.2,137.7,128.3,127.6,127.5$, 96.6, 73.5, 72.9, 70.7, 70.1, 70.0, 69.2, 55.1, 20.8, 20.6; HRMS (ESI, $\mathrm{M}+\mathrm{Na}^{+}$) calcd for $\mathrm{C}_{18} \mathrm{H}_{24} \mathrm{O}_{8} \mathrm{Na} 391.1369$, found 391.1362.

4-Methylphenyl 2,3-di-O-acetyl-6- $O$-benzyl-1-thio- $\beta$-D-glucopyranoside (6b). Prepared according to the general procedure discussed above: yellow oil $(83 \mathrm{mg}, 82 \%) ; R_{\mathrm{f}} 0.25$ (EtOAc/Hex $=$ $1 / 2) ;[\alpha]_{\mathrm{D}}^{26}-37.4$ (c 0.8, DCM); IR ( NaCl) $\nu 3478,3030,2920,1752$, 1494, $1373 \mathrm{~cm}^{-1} ;{ }^{1} \mathrm{H}$ NMR (400 MHz, $\left.\mathrm{CDCl}_{3}\right) \delta 7.40-7.28(\mathrm{~m}$, $7 \mathrm{H}), 7.08(\mathrm{~d}, J=8.4 \mathrm{~Hz}, 2 \mathrm{H}), 5.06(\mathrm{t}, J=9.6 \mathrm{~Hz}, 1 \mathrm{H}), 4.90(\mathrm{dd}, J=$ 9.6, 9.2 Hz, 1H), $4.63(\mathrm{~d}, J=10.0 \mathrm{~Hz}, 1 \mathrm{H}), 4.57(\mathrm{~d}, J=5.2 \mathrm{~Hz}, 2 \mathrm{H})$, 3.80 (dd, $J=6.4,4.8 \mathrm{~Hz}, 2 \mathrm{H}), 3.72(\mathrm{t}, J=9.4 \mathrm{~Hz}, 1 \mathrm{H}), 3.58-3.52$ (m, 1H), $2.32(\mathrm{~s}, 3 \mathrm{H}), 2.09$ (s, 3H), 2.07 (s, 3H).; ${ }^{13} \mathrm{C}$ NMR $(100$ $\left.\mathrm{MHz} \mathrm{CDCl}_{3}\right) \delta 171.2,169.5,138.4,137.6,133.4,129.6,128.4$, 128.0, 127.8, 127.6, 85.8, 78.4, 76.7, 73.7, 70.0, 69.9, 69.87, 21.1, 20.8.; HRMS (ESI, $\mathrm{M}^{+} \mathrm{Na}^{+}$) calcd for $\mathrm{C}_{24} \mathrm{H}_{28} \mathrm{O}_{7} \mathrm{SNa}$ 483.1453, found 483.1446.

4-Methylphenyl 2,3,6-tri- $O$-benzyl-1-thio- $\beta$-D-glucopyranoside (6c). Prepared according to the general procedure discussed above: yellow oil ( $86 \mathrm{mg}, 86 \%) ; R_{\mathrm{f}} 0.25$ (EtOAc/Hex $\left.=1 / 2\right)$; mp 62-64 ${ }^{\circ} \mathrm{C} ;[\alpha]_{\mathrm{D}}^{26}-7.4$ (c 0.8, DCM); IR ( NaCl) $\nu$ 3478, 3030, 2920, 1752, 1494, $1373 \mathrm{~cm}^{-1} ;{ }^{1} \mathrm{H}$ NMR (400 MHz, $\left.\mathrm{CDCl}_{3}\right) \delta 7.46$ (d, $J=8.1 \mathrm{~Hz}, 2 \mathrm{H}), 7.44-7.40(\mathrm{~m}, 2 \mathrm{H}), 7.38-7.27$ (m, 13H), 7.05 $(\mathrm{d}, J=7.9 \mathrm{~Hz}, 2 \mathrm{H}), 4.93(\mathrm{~d}, J=4.4 \mathrm{~Hz}, 1 \mathrm{H}), 4.90(\mathrm{~d}, J=6.0 \mathrm{~Hz}$, $1 \mathrm{H}), 4.76(\mathrm{dd}, J=16.0,15.2 \mathrm{~Hz}, 2 \mathrm{H}), 4.63(\mathrm{~d}, J=9.6 \mathrm{~Hz}, 1 \mathrm{H}), 4.57$ $(\mathrm{d}, J=4.4 \mathrm{~Hz}, 2 \mathrm{H}), 3.77(\mathrm{t}, J=5.0 \mathrm{~Hz}, 2 \mathrm{H}), 3.64(\mathrm{t}, J=9.2 \mathrm{~Hz}$, $1 \mathrm{H}), 3.53(\mathrm{t}, J=8.6 \mathrm{~Hz}, 1 \mathrm{H}), 3.49-3.42(\mathrm{~m}, 2 \mathrm{H}), 2.55(\mathrm{~s}, 1 \mathrm{H}), 2.31$ $(\mathrm{s}, 3 \mathrm{H}) . ;{ }^{13} \mathrm{C}$ NMR $\left(100 \mathrm{MHz}, \mathrm{CDCl}_{3}\right) \delta 138.4,138.2,138.1,138.0$, 137.9, 137.7, 132.6, 129.7, 129.6, 128.6, 128.4, 128.37, 128.2, 127.9, 127.7, 87.9, 86.1, 80.4, 78.0, 75.5, 75.3, 73.6, 71.6, 70.3, 21.1; HRMS (ESI, $\mathrm{M}+\mathrm{Na}^{+}$) calcd for $\mathrm{C}_{34} \mathrm{H}_{36} \mathrm{O}_{5} \mathrm{SNa} 579.2181$, found 579.2184 .

4-Methylphenyl 2,3-di-O-acetyl-6-O-benzyl-1-thio- $\beta$-D-galacopyranoside (6d). Prepared according to the general procedure discussed above: white solid ( $80 \mathrm{mg}, 79 \%) ; R_{\mathrm{f}} 0.50$ (EtOAc/ Hex = 1/1); mp 114-115 ${ }^{\circ} \mathrm{C}$; $[\alpha]_{\mathrm{D}}^{26}+1.8$ (c 0.8, DCM); IR (NaCl) $\nu$ 3478, 3030, 2922, 1750, 1494, $1369 \mathrm{~cm}^{-1}$; ${ }^{1} \mathrm{H}$ NMR $(400 \mathrm{MHz}$, $\left.\mathrm{CDCl}_{3}\right) \delta 7.41(\mathrm{~d}, J=8.0 \mathrm{~Hz}, 2 \mathrm{H}), 7.36-7.29(\mathrm{~m}, 5 \mathrm{H}), 7.07(\mathrm{~d}, J=$ $8.1 \mathrm{~Hz}, 2 \mathrm{H}), 5.27(\mathrm{t}, J=9.8 \mathrm{~Hz}, 1 \mathrm{H}), 4.96(\mathrm{dd}, J=9.6,3.0 \mathrm{~Hz}, 1 \mathrm{H})$, $4.63(\mathrm{~d}, J=10.0 \mathrm{~Hz}, 1 \mathrm{H}), 4.56(\mathrm{~d}, J=4.8 \mathrm{~Hz}, 2 \mathrm{H}), 4.17(\mathrm{t}, J=$ $3.2 \mathrm{~Hz}, 1 \mathrm{H}), 3.78(\mathrm{t}, J=4.4 \mathrm{~Hz}, 2 \mathrm{H}), 3.71(\mathrm{t}, J=5.0 \mathrm{~Hz}, 1 \mathrm{H}), 2.64-$ $2.59(\mathrm{~m}, 1 \mathrm{H}), 2.31(\mathrm{~s}, 3 \mathrm{H}), 2.08(\mathrm{~s}, 3 \mathrm{H}), 2.07$ (s, 3H); ${ }^{13} \mathrm{C}$ NMR $(100$ $\left.\mathrm{MHz}, \mathrm{CDCl}_{3}\right) \delta 170.2,169.5,164.8,155.2,138.3,137.5,133.3$, 129.6, 128.5, 128.3, 127.9, 127.8, 86.5, 76.7, 74.5, 73.8, 69.5, 68.3, 67.6, 29.7, 21.2, 20.9; HRMS (ESI, $\mathrm{M}+\mathrm{Na}^{+}$) calcd for $\mathrm{C}_{24} \mathrm{H}_{28} \mathrm{O}_{7} \mathrm{SNa} 483.1453$, found 483.1458.

3-O-Acetyl-2-azido-6-O-benzyl-2-deoxyl-D-glucopyranoside

(6e). Prepared according to the general procedure discussed above: colorless liquid ( $52 \mathrm{mg}, 69 \%$ ), $\alpha / \beta=3 / 2 ; R_{\mathrm{f}} 0.30$ (EtOAc/ Hex = 1/1); $[\alpha]_{\mathrm{D}}^{25}-5.5$ (c 0.6, DCM); IR (NaCl) $\nu 3417,2923,2871$, 2110, 1720, 1454, $1364 \mathrm{~cm}^{-1}$; ; ${ }^{1} \mathrm{H}$ NMR (400 MHz, $\left.\mathrm{CDCl}_{3}\right) \delta 7.37-$ $7.27(\mathrm{~m}, 8.4 \mathrm{H}), 5.33(\mathrm{~d}, J=10.0 \mathrm{~Hz}, 0.7 \mathrm{H}), 5.30(\mathrm{~d}, J=3.2 \mathrm{~Hz}$, $1 \mathrm{H}), 4.78(\mathrm{dd}, J=10.2,8.7 \mathrm{~Hz}, 0.9 \mathrm{H}), 4.58-4.54(\mathrm{~m}, 3 \mathrm{H}), 4.08$ (ddd, $J=9.2,5.7,2.8 \mathrm{~Hz}, 1 \mathrm{H}), 3.74(\mathrm{t}, J=3.1 \mathrm{~Hz}, 0.8 \mathrm{H}), 3.72(\mathrm{~d}, J$
$=2.8 \mathrm{~Hz}, 0.6 \mathrm{H}), 3.68-3.62(\mathrm{~m}, 2 \mathrm{H}), 3.55(\mathrm{~d}, J=9.5 \mathrm{~Hz}, 1 \mathrm{H}), 3.52-$ $3.49(\mathrm{~m}, 1 \mathrm{H}), 3.34(\mathrm{dd}, J=10.2,8.0 \mathrm{~Hz}, 0.8 \mathrm{H}), 3.28(\mathrm{dd}, J=10.5$, $3.4 \mathrm{~Hz}, 1 \mathrm{H}), 2.16(\mathrm{~s}, 3 \mathrm{H}), 2.15(\mathrm{~s}, 2 \mathrm{H}) ;{ }^{13} \mathrm{C} \mathrm{NMR}\left(100 \mathrm{MHz}, \mathrm{CDCl}_{3}\right)$ $\delta 172.0,171.8,137.3,137.2,128.53,128.5,128.02,127.98,95.9$, 91.9, 75.7, 74.6, 73.7, 73.6, 73.5, 70.5, 70.3, 70.1, 69.5, 69.4, 64.5, 61.4, 21.0; HRMS (ESI, $\mathrm{M}+\mathrm{Na}^{+}$) calcd for $\mathrm{C}_{15} \mathrm{H}_{19} \mathrm{O}_{6} \mathrm{~N}_{3} \mathrm{Na}$ 360.1172 , found 360.1164 .

4-Methylphenyl 3-O-acetyl-6-O-benzyl-2-deoxyl-2-phthalimido-1-thio- $\boldsymbol{\beta}$-D-glucopyranoside (6f). Prepared according to the general procedure discussed above: white solid (159 mg, $80 \%) ; R_{\mathrm{f}} 0.38(\mathrm{EtOAc} / \mathrm{Hex}=1 / 1) ; \mathrm{mp} 54-56{ }^{\circ} \mathrm{C} ;[\alpha]_{\mathrm{D}}^{23}+17.0(c$ 1.0, DCM); IR (NaCl) $\nu ~ 3478,3030,2922,2855,1750,1494$, 1430, $1369 \mathrm{~cm}^{-1} ;{ }^{1} \mathrm{H}$ NMR (400 MHz, $\left.\mathrm{CDCl}_{3}\right) \delta 7.89-7.84(\mathrm{~m}$, $2 \mathrm{H}), 7.77-7.72(\mathrm{~m}, 2 \mathrm{H}), 7.40-7.31(\mathrm{~m}, 5 \mathrm{H}), 7.29(\mathrm{~d}, J=8.2 \mathrm{~Hz}$, $2 \mathrm{H}), 7.03(\mathrm{~d}, J=7.9 \mathrm{~Hz}, 2 \mathrm{H}), 5.68-5.61(\mathrm{~m}, 2 \mathrm{H}), 4.60(\mathrm{q}, J=$ 11.8 Hz, 2H), 4.26 (t, $J=10.4 \mathrm{~Hz}, 1 \mathrm{H}), 3.88-3.72(\mathrm{~m}, 4 \mathrm{H}), 2.91$ (s, 1H), $2.29(\mathrm{~s}, 3 \mathrm{H}), 1.91(\mathrm{~s}, 3 \mathrm{H}) ;{ }^{13} \mathrm{C} \mathrm{NMR}\left(100 \mathrm{MHz}, \mathrm{CDCl}_{3}\right)$ $\delta 171.1,167.8,167.3,138.4,137.7,134.3,134.1,133.5,131.6$, $131.2,129.6,128.4,127.8,127.7,127.5,123.6,123.5,83.2$, 78.3, 74.3, 73.7, 71.0, 70.2, 53.6, 52.6, 21.1, 20.7, 20.5, 13.9; HRMS (ESI, $\mathrm{M}+\mathrm{Na}^{+}$) calcd for $\mathrm{C}_{30} \mathrm{H}_{29} \mathrm{NO}_{7} \mathrm{SNa} 570.1562$, found 570.1561 .

\section{Glycosylation reactions}

2-Azido-2-deoxy-3-O-acetyl-6-O-benzyl-1-O-tert-butyldimethylsilyl-4-O-(3-O-ace-tyl-4,6-O-benzylidene-2-deoxy-2-phthalimido- $\beta$-D-glucopyranosyl)- $\boldsymbol{\beta}$-D-glucopyranoside (13). A solution of acceptor $6 \mathrm{~g}$ (50 mg, $0.11 \mathrm{mmol})$, donor $5 \mathrm{f}$ (120 mg, 0.22 mmol), and activated $4 \AA$ molecular sieves (300 mg) in dichloromethane $(1 \mathrm{~mL})$ was stirred for 30 minutes at room temperature. After $N$-iodosuccinimide (148 $\mathrm{mg}, 0.66 \mathrm{mmol}$ ) and dried phosphotungstic acid (158 $\mathrm{mg}, 0.055 \mathrm{mmol}$ ) were added, the reaction mixture was stirred for 5 hours. When the reaction was completed, molecular sieves were removed by filtration through Celite. The filtrate was extracted with aqueous sodium thiosulfate $(10 \mathrm{~mL})$ and brine $(10 \mathrm{~mL})$, and the organic layer was dried over anhydrous $\mathrm{MgSO}_{4}$, filtered, and concentrated under vacuum. The residue was purified by column chromatography on silica gel to give the desired product $13(79 \mathrm{mg}, 82 \%)$ as a white soild. $R_{\mathrm{f}} 0.47$ (EtOAc/Hex $=1 / 2) ; \operatorname{mp} 112-114{ }^{\circ} \mathrm{C} ;[\alpha]_{\mathrm{D}}^{24}-13.4$ (c 1.0, DCM); IR (NaCl) $\nu$ 3479, 2931, 2112, 1751, 1720, 1386, 1227, 1105, $1082 \mathrm{~cm}^{-1}$; ${ }^{1} \mathrm{H}$ NMR (400 MHz, $\left.\mathrm{CDCl}_{3}\right) \delta 7.83(\mathrm{dd}, J=5.5,3.1 \mathrm{~Hz}, 2 \mathrm{H})$, $7.71(\mathrm{dd}, J=5.5,3.0 \mathrm{~Hz}, 2 \mathrm{H}), 7.46(\mathrm{~d}, J=4.4 \mathrm{~Hz}, 1 \mathrm{H}), 7.44(\mathrm{~d}, J$ $=2.1 \mathrm{~Hz}, 1 \mathrm{H}), 7.38-7.35(\mathrm{~m}, 3 \mathrm{H}), 7.32-7.27(\mathrm{~m}, 3 \mathrm{H}), 7.21-$ $7.19(\mathrm{~m}, 2 \mathrm{H}), 5.84(\mathrm{dd}, J=10.2,9.4 \mathrm{~Hz}, 1 \mathrm{H}), 5.52(\mathrm{~s}, 1 \mathrm{H}), 5.50$ $(\mathrm{d}, J=8.4 \mathrm{~Hz}, 1 \mathrm{H}), 4.90(\mathrm{dd}, J=10.4,9.2 \mathrm{~Hz}, 1 \mathrm{H}), 4.47(\mathrm{~d}, J=$ $8.0 \mathrm{~Hz}, 1 \mathrm{H}), 4.39-4.36(\mathrm{~m}, 2 \mathrm{H}), 4.26(\mathrm{~d}, J=12.0 \mathrm{~Hz}, 1 \mathrm{H}), 4.21$ (dd, $J=10.4,8.4 \mathrm{~Hz}, 1 \mathrm{H}), 3.93(\mathrm{t}, J=9.6 \mathrm{~Hz}, 1 \mathrm{H}), 3.79-3.70$ $(\mathrm{m}, 2 \mathrm{H}), 3.65-3.59(\mathrm{~m}, 1 \mathrm{H}), 3.36-3.33(\mathrm{~m}, 1 \mathrm{H}), 3.30-3.25(\mathrm{~m}$, $3 \mathrm{H}), 2.15(\mathrm{~s}, 3 \mathrm{H}), 1.86(\mathrm{~s}, 3 \mathrm{H}), 0.88(\mathrm{~s}, 9 \mathrm{H}), 0.07$ (s, 3H), 0.07 $(\mathrm{s}, 3 \mathrm{H}) ;{ }^{13} \mathrm{C}$ NMR $\left(101 \mathrm{MHz}, \mathrm{CDCl}_{3}\right) \delta 170.1,169.2,137.8$, 136.6, 134.2, 131.2, 129.1, 128.1, 127.4, 127.3, 126.1, 123.4, 101.5, 98.2, 96.7, 78.8, 74.6, 74.2, 72.7, 72.4, 69.5, 68.5, 67.3, $66.3,65.8,55.5,25.4,21.1,20.4,17.8,-4.6,-5.4$; HRMS (ESI, $\mathrm{M}+\mathrm{Na}^{+}$) calcd for $\mathrm{C}_{44} \mathrm{H}_{52} \mathrm{~N}_{4} \mathrm{O}_{13} \mathrm{SiNa} 895.31978$, found 895.3181. 
2-Azido-4-O-benzyl-1-O-tert-butyldimethylsilyl-2-deo-xy-3-O(2-naphthylmethyl)-6-O-(3-O-acetyl-4,6-O-be-nzylidene-2-deoxy2-phthalimi-do- $\beta$-D-glucopyranosyl)- $\beta$-D-glucopyranoside (14). To a solution of acceptor 12 (241 $\mathrm{mg}, 0.44 \mathrm{mmol})$, donor $\mathbf{5 f}$ (200 mg, $0.37 \mathrm{mmol}$ ), $N$-iodosuccinimide (90 mg, $0.40 \mathrm{mmol}$ ) and activated $4 \AA$ molecular sieves $(150 \mathrm{mg})$ in dichloromethane $(3.0 \mathrm{~mL})$ were stirred for 1 hour at room temperature. After phosphotungstic acid (126 mg, $0.044 \mathrm{mmol}$ ) was added at $0{ }^{\circ} \mathrm{C}$, the reaction mixture was stirred for 12 hours at same temperature. When the reaction was completed, the mixture was quenched by triethylamine and molecular sieves were removed by filtration through Celite. The filtrate was extracted with aqueous sodium thiosulfate $(10 \mathrm{~mL})$ and brine $(10 \mathrm{~mL})$, and the organic layer was dried over anhydrous $\mathrm{MgSO}_{4}$, filtered, and concentrated under vacuum. The residue was purified by column chromatography on silica gel to give the desired product 14 (84 mg, 53\%) as pali yellow soild. $R_{\mathrm{f}} 0.50$ (EtOAc/Hex $=1 / 2) ; \operatorname{mp~90-93~}{ }^{\circ} \mathrm{C} ;[\alpha]_{\mathrm{D}}^{25}-93.63$ (c 0.1, DCM); IR (NaCl) v 3414, 2106, 1748, 1718, $1645 \mathrm{~cm}^{-1} ;{ }^{1} \mathrm{H}$ NMR (400 MHz, $\left.\mathrm{CDCl}_{3}\right) \delta 7.86-$ $7.79(\mathrm{~m}, 3 \mathrm{H}), 7.75-7.72(\mathrm{~m}, 3 \mathrm{H}), 7.60-7.58(\mathrm{~m}, 2 \mathrm{H}), 7.51-7.45$ $(\mathrm{m}, 5 \mathrm{H}), 7.39(\mathrm{~d}, J=4.4 \mathrm{~Hz}, 3 \mathrm{H}), 7.28-7.24(\mathrm{~m}, 4 \mathrm{H}), 7.03(\mathrm{~d}, J=$ $6.0 \mathrm{~Hz}, 2 \mathrm{H}), 5.87(\mathrm{t}, J=10 \mathrm{~Hz}, 1 \mathrm{H}), 5.57(\mathrm{~s}, 1 \mathrm{H}), 5.53(\mathrm{~d}, J=8.4$, $1 \mathrm{H}), 5.00(\mathrm{~d}, J=11.2 \mathrm{~Hz}, 1 \mathrm{H}), 4.86(\mathrm{~d}, J=11.2 \mathrm{~Hz}, 1 \mathrm{H}), 4.60(\mathrm{~d}, J$ $=10.8 \mathrm{~Hz}, 1 \mathrm{H}), 4.39(\mathrm{t}, J=8,2 \mathrm{H}), 4.32(\mathrm{~d}, J=10.8 \mathrm{~Hz}, 1 \mathrm{H}), 4.23$ $(\mathrm{d}, J=10 \mathrm{~Hz}, 1 \mathrm{H}), 3.88-3.79(\mathrm{~m}, 2 \mathrm{H}), 3.77-3.69(\mathrm{~m}, 2 \mathrm{H}), 3.46-$ $3.36(\mathrm{~m}, 2 \mathrm{H}), 3.34-3.29$ (m, 2H), 1.90 (s, 3H), 0.89 (s, 9H), 0.08 (s, $3 \mathrm{H}), 0.00(\mathrm{~s}, 3 \mathrm{H}) ;{ }^{13} \mathrm{C}$ NMR (100 $\left.\mathrm{MHz}, \mathrm{CDCl}_{3}\right) \delta 170.0,137.4$, $136.8,135.3$, 134.0, 133.1, 132.8, 129.0, 128.2, 128.1, 128.0, $127.8,127.6,127.5,127.5,126.5,126.1,125.9,125.9,125.8$, 123.3, 101.5, 98.0, 97.0, 82.4, 79.0, 77.5, 75.2, 74.7, 74.1, 69.8, $68.5,68.3,67.9,66.1,55.0,30.7,25.4,20.4,17.7,-4.4,-5.6$; HRMS (ESI, $\mathrm{M}+\mathrm{Na}^{+}$) calcd for $\mathrm{C}_{53} \mathrm{H}_{58} \mathrm{~N}_{4} \mathrm{O}_{12} \mathrm{SiNa}$ 993.3718, found 993.3707.

2-Azido-2-deoxy-3-O-acetyl-4-O-benzyl-1-O-tert-butyldimethylsilyl-6-O-(3-O-ace-tyl-4,6-O-benzylidene-2-phthalimido-2deoxy- $\beta$-D-glucopyranosyl)- $\beta$-D-gluco-pyranoside (15). To a solution of acceptor 11 (99 mg, $0.22 \mathrm{mmol})$, donor $\mathbf{5 f}$ (100 mg, 0.18 mmol), $N$-iodosuccinimide (61 $\mathrm{mg}, 0.27 \mathrm{mmol}$ ) and activated 4 $\AA$ molecular sieves $(200 \mathrm{mg})$ in dichloromethane $(1.5 \mathrm{~mL})$ were stirred for 1 hour at room temperature. After dried phosphotungstic acid (158 $\mathrm{mg}, 0.054 \mathrm{mmol}$ ) was added, the reaction mixture was stirred for 3 hours at $-40{ }^{\circ} \mathrm{C}$. When the reaction was completed, it was quenched by triethylamine and molecular sieves were removed by filtration through Celite. The filtrate was extracted with aqueous sodium thiosulfate $(10 \mathrm{~mL})$ and brine $(10 \mathrm{~mL})$, and the organic layer was dried over anhydrous $\mathrm{MgSO}_{4}$, filtered, and concentrated under vacuum. The residue was purified by column chromatography on silica gel to give the desired product $15(84 \mathrm{mg}, 53 \%) . R_{\mathrm{f}} 0.30($ EtOAc/Hex $=$ 1.5/8.5); mp $175-176{ }^{\circ} \mathrm{C}$; $[\alpha]_{\mathrm{D}}^{25}-152.2$ (c 1.0, DCM); IR (NaCl) $v$ 2951, 2931, 2884, 2858, 2111, 1747, 1718, 1645, 1469, 1387, 1312, 1225, 1176, 1104, 1042, 1014, 1001, 970, 917, 872, 840, 784, 738, $700 \mathrm{~cm}^{-1} ;{ }^{1} \mathrm{H}$ NMR (400 $\left.\mathrm{MHz} \mathrm{CDCl}_{3}\right) \delta 7.76(\mathrm{~s}, 2 \mathrm{H})$, 7.65-7.63 (m, 2H), 7.47-7.44 (m, 2H), 7.37-7.35 (m, 3H), 7.22$7.21(\mathrm{~m}, 3 \mathrm{H}), 6.96-6.94(\mathrm{~m}, 2 \mathrm{H}), 5.84(\mathrm{t}, J=9.2 \mathrm{~Hz}, 1 \mathrm{H}), 5.54(\mathrm{~s}$, $1 \mathrm{H}), 5.51(\mathrm{~d}, J=8.4 \mathrm{~Hz}, 1 \mathrm{H}), 4.91(\mathrm{t}, J=8.8 \mathrm{~Hz}, 1 \mathrm{H}), 4.54(\mathrm{~d}, J=$
$9.2 \mathrm{~Hz}, 1 \mathrm{H}), 4.41-4.35(\mathrm{~m}, 2 \mathrm{H}), 4.27(\mathrm{~d}, J=4 \mathrm{~Hz}, 2 \mathrm{H}), 4.00(\mathrm{~d}, J=$ $10.4 \mathrm{~Hz}, 1 \mathrm{H}), 3.86-3.76(\mathrm{~m}, 2 \mathrm{H}), 3.75-3.69$ (m, 2H), 3.48-3.37 $(\mathrm{m}, 2 \mathrm{H}), 3.22(\mathrm{dd}, J=12.8,8.8 \mathrm{~Hz}, 1 \mathrm{H}), 1.97(\mathrm{~s}, 3 \mathrm{H}), 1.89(\mathrm{~s}, 3 \mathrm{H})$, 0.86 (S, 9H), 0.07 (s, 3H), 0.01 (s, 3H); ${ }^{13} \mathrm{C}$ NMR (100 MHz, $\left.\mathrm{CDCl}_{3}\right) \delta 170.1,169.7,137.1,136.8,134.2,129.1,128.3,128.2$, 127.8, 127.5, 126.2, 123.5, 101.6, 98.1, 97.0, 79.1, 76.0, 74.3, 74.0, 73.5, 69.8, 68.6, 67.8, 66.3, 66.2, 55.1, 25.4, 20.8, 20.5, 17.8, -4.3, -5.4; HRMS (ESI, $\mathrm{M}+\mathrm{Na}^{+}$) calcd for $\mathrm{C}_{44} \mathrm{H}_{52} \mathrm{~N}_{4} \mathrm{O}_{13} \mathrm{SiNa} 895.3197$, found 895.3193.

2-Azido-4,6-O-benzylidene-1-O-tert-butyldimethylsilyl-2deoxyl-3-O-(3-O-acetyl-4,6-O-benzylidene-2-deoxyl-2-phtha-limido- $\beta$-D-glucopyranosyl)- $\beta$-D-glucopyranoside (16). A solution of acceptor $4 \mathrm{c}(100 \mathrm{mg}, 0.25 \mathrm{mmol})$ and dried phosphotungstic acid (177 mg, $0.062 \mathrm{mmol})$ in dichloromethane $(2.68 \mathrm{~mL}$ ) was stirred for 30 minutes at $28{ }^{\circ} \mathrm{C}$ with activated $4 \AA$ molecular sieves (335 mg). Then $N$-iodosuccinimide (166 mg, $0.74 \mathrm{mmol}$ ) and donor $5 \mathbf{f}(235 \mathrm{mg}, 0.43 \mathrm{mmol})$ in dichloromethane $(4 \mathrm{~mL})$ was added, and the reaction mixture was stirred for 8 hours. When the reaction was completed, molecular sieves were removed by filtration through Celite. The filtrate was extracted with aqueous sodium thiosulfate $(20 \mathrm{~mL})$ and brine $(20 \mathrm{~mL})$, and the organic layers were dried over anhydrous $\mathrm{MgSO}_{4}$, filtered, and concentrated under vacuum. The residue was purified by column chromatography on silica gel to give the desired product $16(176 \mathrm{mg}, 86 \%)$ as a white soild. $R_{\mathrm{f}} 0.52$ $($ EtOAc/Hex $=1 / 2) ; \operatorname{mp} 260-262{ }^{\circ} \mathrm{C} ;[\alpha]_{\mathrm{D}}^{24}-64.3(c 1.0, \mathrm{DCM}) ; \mathrm{IR}$ $(\mathrm{NaCl}) \nu 3441,2931,2112,1745,1719,1387,1225,1098 \mathrm{~cm}^{-1} ;{ }^{1} \mathrm{H}$ NMR (400 MHz, $\left.\mathrm{CDCl}_{3}\right) \delta 7.85(\mathrm{dd}, J=5.2,3.0 \mathrm{~Hz}, 2 \mathrm{H}), 7.68(\mathrm{dd}$, $J=5.4,3.0 \mathrm{~Hz}, 2 \mathrm{H}), 7.49-7.43(\mathrm{~m}, 4 \mathrm{H}), 7.39-7.34(\mathrm{~m}, 6 \mathrm{H}), 5.90(\mathrm{t}$, $J=9.8 \mathrm{~Hz}, 1 \mathrm{H}), 5.56(\mathrm{~d}, J=8.4 \mathrm{~Hz}, 1 \mathrm{H}), 5.50(\mathrm{~d}, J=14.8 \mathrm{~Hz}, 2 \mathrm{H})$, $4.53(\mathrm{~d}, J=7.6 \mathrm{~Hz}, 1 \mathrm{H}), 4.39$ (dd, $J=10.2,8.6 \mathrm{~Hz}, 1 \mathrm{H}), 4.24$ (dd, $J$ $=10.6,5.0 \mathrm{~Hz}, 1 \mathrm{H}), 4.07(\mathrm{dd}, J=10.0,4.4 \mathrm{~Hz}, 1 \mathrm{H}), 3.78-3.69(\mathrm{~m}$, $3 \mathrm{H}), 3.66(\mathrm{dd}, J=9.4,4.2 \mathrm{~Hz}, 1 \mathrm{H}), 3.58(\mathrm{t}, J=9.2 \mathrm{~Hz}, 1 \mathrm{H}), 3.48(\mathrm{t}$, $J=9.4 \mathrm{~Hz}, 1 \mathrm{H}), 3.31(\mathrm{td}, J=9.6,5.0 \mathrm{~Hz}, 1 \mathrm{H}), 3.20(\mathrm{dd}, J=9.6$, $7.6 \mathrm{~Hz}, 1 \mathrm{H}), 1.88(\mathrm{~s}, 3 \mathrm{H}), 0.85(\mathrm{~s}, 9 \mathrm{H}), 0.07$ (s, 3H), $0.06(\mathrm{~s}, 3 \mathrm{H})$; ${ }^{13} \mathrm{C}$ NMR $\left(101 \mathrm{MHz}, \mathrm{CDCl}_{3}\right) \delta 170.0,136.9,136.8,134.1,131.3$, 129.0, 128.1, 128.0, 126.2, 125.9, 123.4, 101.5, 101.1, 99.8, 97.7, 80.6, 79.0, 78.9, 69.7, 68.5, 68.2, 67.4, 66.3, 66.0, 55.4, 25.4, 20.5, 17.7, -4.5, -5.3; HRMS (ESI, $\mathrm{M}+\mathrm{H}^{+}$) calcd for $\mathrm{C}_{42} \mathrm{H}_{49} \mathrm{~N}_{4} \mathrm{O}_{12} \mathrm{Si}$ 829.3116 , found 829.3113 .

2-Azido-4,6-O-benzylidene-1-O-tert-butyldimethylsilyl-2deoxyl-3- $O$-(2,3,4,6-tetra- $O$-acetyl- $\beta$-D-glucopyranosyl)- $\beta$-D-glucopyranoside (17). A solution of acceptor $4 \mathbf{c}(100 \mathrm{mg}, 0.25 \mathrm{mmol})$ and donor 10 (167 mg, $0.37 \mathrm{mmol})$ in dichloromethane $(5.5 \mathrm{~mL})$ was stirred for 30 minutes at room temperature with activated 4 $\AA$ molecular sieves (323 mg). After $N$-iodosuccinimide (166 mg, $0.74 \mathrm{mmol}$ ) and phosphotungstic acid (216 $\mathrm{mg}, 0.075 \mathrm{mmol}$ ) were added, the reaction mixture was stirred for 5 hours at room temperature. When the reaction was completed, molecular sieves were removed by filtration through Celite. The filtrate was extracted with aqueous sodium thiosulfate $(10 \mathrm{~mL})$ and brine $(10 \mathrm{~mL})$, and the organic layers were dried over anhydrous $\mathrm{MgSO}_{4}$, filtered, and concentrated under vacuum. The residue was purified by column chromatography on silica gel to give the desired product $17(123 \mathrm{mg}, 67 \%)$ as a white soild. $R_{\mathrm{f}} 0.51$ $($ EtOAc/Hex $=3 / 4)$; mp 96-98 ${ }^{\circ} \mathrm{C} ;[\alpha]_{\mathrm{D}}^{25}-40.4$ (c 1.0, DCM); IR $(\mathrm{NaCl}) \nu$ 3472, 2933, 2860, 2114, 1757, 1371, 1228, 1099, 
$1040 \mathrm{~cm}^{-1} ;{ }^{1} \mathrm{H}$ NMR $\left(400 \mathrm{MHz}, \mathrm{CDCl}_{3}\right) \delta 7.44(\mathrm{dd}, J=6.8,2.9 \mathrm{~Hz}$, $2 \mathrm{H}), 7.33-7.32(\mathrm{~m}, 3 \mathrm{H}), 5.52(\mathrm{~s}, 1 \mathrm{H}), 5.16(\mathrm{~d}, J=9.6 \mathrm{~Hz}, 1 \mathrm{H})$, 5.09-5.00 (m, 2H), $4.74(\mathrm{~d}, J=8.0 \mathrm{~Hz}, 1 \mathrm{H}), 4.59(\mathrm{~d}, J=8.0 \mathrm{~Hz}$, $1 \mathrm{H}), 4.25(\mathrm{dd}, J=10.6,5.0 \mathrm{~Hz}, 1 \mathrm{H}), 4.08(\mathrm{dd}, J=12.4,4.0 \mathrm{~Hz}$, $1 \mathrm{H}), 3.84(\mathrm{dd}, J=12.4,2.4 \mathrm{~Hz}, 1 \mathrm{H}), 3.77$ (t, $J=10.4 \mathrm{~Hz}, 1 \mathrm{H}), 3.66$ $(\mathrm{t}, J=9.2 \mathrm{~Hz}, 1 \mathrm{H}), 3.59(\mathrm{t}, J=9.4 \mathrm{~Hz}, 1 \mathrm{H}), 3.47-3.43(\mathrm{~m}, 1 \mathrm{H}), 3.36$ $(\mathrm{dd}, J=9.6,4.8 \mathrm{~Hz}, 1 \mathrm{H}), 3.31$ (dd, $J=9.6,7.6 \mathrm{~Hz}, 1 \mathrm{H}), 2.05$ (s, $3 \mathrm{H}), 1.98$ (s, 3H), 1.97 (s, 3H), $1.96(\mathrm{~s}, 3 \mathrm{H}), 0.91$ (s, 9H), 0.15 (s, $3 \mathrm{H}), 0.13(\mathrm{~s}, 3 \mathrm{H}) ;{ }^{13} \mathrm{C} \mathrm{NMR}\left(101 \mathrm{MHz}, \mathrm{CDCl}_{3}\right) \delta 170.5,170.2$, 169.3, 169.2, 136.9, 129.1, 128.1, 125.9, 101.2, 101.0, 97.5, 79.4, 79.1, 72.7, 71.6, 71.4, 68.4, 68.3, 67.9, 66.4, 61.4, 25.4, 20.6, 20.5, 20.5, 17.8, -4.5, -5.3; HRMS (ESI, $\mathrm{M}+\mathrm{Na}^{+}$) calcd for $\mathrm{C}_{33} \mathrm{H}_{47^{-}}$ $\mathrm{N}_{3} \mathrm{O}_{14} \mathrm{SiNa} 760.2725$, found 760.2732 .

\section{Author contribution}

S.-Y. L. designed the research. J.-S. C. and S.-S. W. prepared compounds 2a-2f of Table 1. A. S. prepared compounds $\mathbf{4 a - 4 e}$ of Table 2. J.-S. C., P.-H. H., and C.-H. L. prepared compounds 6a-6f of Table 3. Y.-J. L. and A. S. prepared compounds 13-17 of Table 4. H. R. W. analyzed the Mass data. A. S. and S.-Y. L. wrote the manuscript.

\section{Conflicts of interest}

There are no conflicts to declare.

\section{Acknowledgements}

The authors thank the Ministry of Science and Technology (MOST) in Taiwan (MOST 107-2113-M-005-021) and National Chung Hsing University for financial support.

\section{Notes and references}

1 (a) C. Pifferi, G. C. Daskhan, M. Fiore, T. C. Shiao, R. Roy and O. Renaudet, Chem. Rev., 2017, 117, 9839; (b) R. J. Wood, Chem. Rev., 2018, 118, 8005; (c) A. Giannis, Angew. Chem., Int. Ed., 1994, 33, 178; (d) A. Varki, Glycobiology, 1993, 3, 97; (e) C. C. Yua and S. G. Withers, Adv. Synth. Catal., 2015, 357, 1633.

2 (a) P. Sears and C. H. Wong, Science, 2001, 291, 2344; (b) M. Kilcoyne and L. Joshi, Cardiovasc. Hematol. Agents Med. Chem., 2007, 5, 186; (c) Y. Chen, M. J. Heeg, P. G. Braunschweiger, W. Xie and P. G. Wang, Angew. Chem., Int. Ed., 1999, 38, 1768; (d) M. C. Galan, D. BenitoAlifonso and G. M. Watt, Org. Biomol. Chem., 2011, 9, 3598; (e) E. Borges de Melo, A. da Silveira Gomes and I. Carvalho, Tetrahedron, 2006, 62, 10277.

3 (a) K. C. Nicolaou and J. M. Helen, Angew. Chem., Int. Ed., 2001, 40, 1576; (b) C. Damiano and L. Luigi, Synlett, 2014, 25, 2873; (c) M. Petitou, P. Duchaussoy, P. A. Driguez, G. Jaurand, J. P. Herault, J. C. Lormeau, C. A. A. van Boeckel and J. M. Herbert, Angew. Chem., Int. Ed., 1998, 37, 3009; (d) R. Dureau, L. Legentil, R. Daniellou and V. Ferrières, J. Org. Chem., 2012, 77, 1301.
4 C. C. Wang, J. C. Lee, S. Y. Luo, S. S. Kulkarni, Y. W. Huang, C. C. Lee, K. L. Chang and S. C. Hung, Nature, 2007, 446, 896. 5 (a) H. Geyer and R. Geyer, Biochim. Biophys. Acta, Proteins Proteomics, 2006, 1764, 1853; (b) S. S. Kulkarni, C. C. Wang, N. M. Sabbavarapu, A. R. Podilapu, P. H. Liao and S. C. Hung, Chem. Rev., 2018, 118, 8025; (c) O. Plettenburg, C. Mui, B. N. Vera and C. H. Wonga, Adv. Synth. Catal., 2002, 344, 622.

6 (a) Z. Zhang, I. R. Ollmann, X. S. Ye, R. Wischnat, T. Baasov and C. H. Wong, J. Am. Chem. Soc., 1999, 121, 734; (b) P. Wang, J. Zhu, Y. Yuan and S. J. Danishefsky, J. Am. Chem. Soc., 2009, 131, 16669; (c) U. Kazmaier, Angew. Chem., Int. Ed., 2005, 44, 2186; (d) S. R. Sanapala and S. S. Kulkarni, J. Am. Chem. Soc., 2016, 138, 4938; (e) N. L. Douglas, S. V. Ley, U. Lucking and S. L. Warriner, J. Chem. Soc., Perkin Trans. 1, 1998, 51.

7 (a) T. J. Boltje, T. Buskas and G. J. Boons, Nat. Chem., 2009, 1, 611; (b) Y. Vohra, V. Mahalakshmi, A. Venot and G. J. Boons, Org. Lett., 2008, 10, 3247.

8 (a) A. T. Tran, R. A. Jones, J. Pastor, J. Boisson, N. Smith and M. C. Galan, Adv. Synth. Catal., 2011, 353, 2593; (b) J. T. Smoot and A. V. Demchenko, Adv. Carbohydr. Chem. Biochem., 2009, 62, 161; (c) M. A. Witsch and G. H. Jacquelyn, Org. Lett., 2010, 12, 4312.

9 (a) C. A. Tai, S. S. Kulkarni and S. C. Hung, J. Org. Chem., 2003, 68, 8719; (b) J. A. Hyatt and G. W. Tindall, Heterocycles, 1993, 35, 227.

10 (a) F. Dasgupta, P. P. Singh and H. C. Srivastava, Carbohydr. Res., 1980, 80, 346; (b) C. T. Chen, J. H. Kuo, C. H. Li, N. B. Barhate, S. W. Hon, T. W. Li, S. D. Chao, C. C. Liu, Y. C. Li, I. H. Chang, J. S. Lin, C. J. Liu and Y. C. Chou, Org. Lett., 2001, 3, 3729.

11 (a) G. Huang, H. Gao, C. Yi, Y. Ai and D. Huang, Pharm. Chem. J., 2017, 51, 111; (b) Y. L. Yan, J. R. Guo and C. F. Liang, Chem.-Asian J., 2017, 12, 2471; (c) T. W. Lin, A. K. Adak, H. J. Lin, A. Das, W. C. Hsiao, T. C. Kuan and C. C. Lin, $R S C A d v ., 2016,6,58749$; (d) B. Yu, J. Xie, S. Deng and Y. Hui, J. Am. Chem. Soc., 1999, 121, 12196.

12 (a) P. J. Garegg, in Preparative Carbohydrate Chemistry, ed. S. Hanessian, 1st edn, 1997, pp. 53-67; (b) A. V. Demchenko, P. Pornsuriyasak and C. D. Meo, J. Chem. Educ., 2006, 83, 782; (c) T. Yamanoi, T. Akiyama, E. Ishida, H. Abe, M. Amemiya and T. Inazu, Chem. Lett., 1989, 18, 335; (d) G. Tanabe, K. Yoshikai, T. Hatanaka, M. Yamamoto, Y. Shao, T. Minematsu, O. Muraoka, T. Wang, H. Matsuda and M. Yoshikawa, Bioorg. Med. Chem., 2007, 15, 3926; (e) S. Y. Han, M. M. Joullié, N. A. Petasis, J. Bigorra, J. Cobera, J. Font and R. M. Ortuño, Tetrahedron, 1993, 49, 349; $(f)$ M. Tatina, S. K. Yousuf and D. Mukherjee, Org. Biomol. Chem., 2012, 10, 5357; (g) R. Panchadhayee and A. K. Mishra, Synthesis, 2010, 64, 1193; (h) M. P. DeNinno, J. B. Etienne and K. C. Duplantier, Tetrahedron Lett., 1995, 36, 669; (i) T. Eyles, J. Richard, D. G. Benjamin and F. J. Antony, Tetrahedron: Asymmetry, 2003, 14, 1201.

13 P. J. Garegg, H. Hultberg and S. Wallin, Carbohydr. Res., 1982, 108, 97. 
14 (a) C. R. Shie, Z. H. Tzeng, S. S. Kulkarni, B. J. Uang, C. Y. Hsu and S. C. Hung, Angew. Chem., 2005, 117, 1693; (b) M. Sakagani and H. Hamana, Tetrahedron Lett., 2000, 41, 5547.

15 (a) H. Guo, W. Si, J. Li, G. Yang, T. Tang, Z. Wang, J. Tang and J. Zhang, Synthesis, 2019, 51, 2984; (b) A. Kitowski, J. M. Ester, M. Salvado, J. Mestre, S. Castillon, J. O. Gonzado, O. Boutureira and G. J. L. Bernardes, Org. Lett., 2017, 19, 5490; (c) Q. Tian, L. Dong, X. Ma, L. Xu, C. Hu, W. Zou and H. Shao, J. Org. Chem., 2011, 76, 1045.

16 (a) M. N. Noshi, e-EROS Encyclopedia of Reagents for Organic Synthesis, 2013, pp. 1-8; (b) L. T. Aany Sofia, A. Krishnan, M. Sankar, N. K. Kala Raj, P. Manikandan, P. R. Rajamohanan and T. G. Ajithkumar, J. Phys. Chem., 2009, 113, 21114; (c) S. Wang, Z. Zhang, B. Liu and J. Li, Catal. Sci. Technol., 2013, 3, 2104.

17 (a) M. Zendehdel, A. Mobinikhaledi, H. Alikhani and N. Jafari, J. Chin. Chem. Soc., 2010, 57, 683; (b) L. S. Gadekar, S. R. Mane, S. S. Katkar, B. R. Arbad and M. K. Lande, Cent. Eur. J. Chem., 2009, 7, 550.

18 (a) F. Marme, G. Condurier and J. C. Vedrine, Microporous Mesoporous Mater., 1998, 22, 151; (b) M. M. Heravi and S. Sadjadi, J. Iran. Chem. Soc., 2009, 6, 1.

19 S. Zhu, X. Gao, F. Dong, Y. Zhu, H. Zheng and Y. Li, J. Catal., 2013, 306, 155.

20 V. R. F. Enrique, C. Pieters, V. D. L. Bart, J. A. Jana, S. C. Pablo, M. W. G. M. Verhoeven, H. Niemantsverdriet, J. Gascon and F. Kapteijn, J. Catal., 2012, 289, 42.

21 M. Dabiri and S. Bashiribod, Molecules, 2009, 14, 1126.
22 (a) W. Zhang, Y. Leng, P. Zhao, J. Wang, D. Zhu and J. Huang, Green Chem., 2011, 13, 832; (b) S. Siddiqui, M. U. Khan and Z. N. Siddiqui, ACS Sustainable Chem. Eng., 2017, 5, 7932.

23 V. U. Vernekar, K. M. Hosamani, I. N. Shaikh and K. C. S. Achar, Arabian J. Chem., 2016, 9, S663.

24 B. Torok, I. T. Bucsi, T. Beregszaszi, I. Kapocsi and A. Molnar, J. Mol. Catal. A: Chem., 1996, 107, 305.

25 Y. Izumi and T. Fujita, J. Mol. Catal. A: Chem., 1996, 106, 43. 26 (a) M. A. Betiha, H. M. A. Hassan, E. A. E. Sharkawy, A. M. A. Sabagh, M. F. Menoufya and H. E. M. Abdelmoniem, Appl. Catal., B, 2016, 182, 15; (b) Z. J. Lin, H. Q. Zheng, J. Chen, W. E Zhuang, Y. X. Lin, J. W. Su, Y. B. Huang and R. Cao, Inorg. Chem., 2018, 57, 13009; (c) T. Zillillah, A. Ngu and Z. Li, Green Chem., 2014, 16, 1202; (d) J. Feng, M. Li and X. Meng, Catal. Lett., 2019, 149, 1504.

27 V. V. Kouznetsov, L. Y. V. Mendez and C. M. M. Gomez, Curr. Org. Chem., 2005, 9, 141.

28 (a) M. Misono and N. Nojiri, Appl. Catal., 1990, 64, 1; (b) Y. Zhang, V. Degirmenci, C. Li and E. J. M. Hensen, ChemSusChem, 2011, 4, 59.

29 I. C. Wei, N. Tsao, Y. H. Huang, Y. S. Ho, C. C. Wu, D. F. Yu and D. J. Yang, Appl. Radiat. Isot., 2008, 66, 320.

30 (a) B. Santhanam and G. J. Boons, Org. Lett., 2004, 6, 3333; (b) Y. Zhang, J. Gaekwad, M. A. Wolfert and G. J. Boons, J. Am. Chem. Soc., 2007, 129, 5200.

31 J. Dinkelaar, H. Gold, H. S. Overkleeft, J. D. C. Codée and G. A. V. D. Marel, J. Org. Chem., 2009, 74, 4208. 